Canad. J. Math. Vol. 71 (1), 2019 pp. 131-152

http://dx.doi.org/10.4153/CJM-2017-048-5

(c) Canadian Mathematical Society 2018. This is an Open Access article, distributed

under the terms of the Creative Commons Attribution licence

(http://creativecommons.org/licenses/by/4.0), which permits unrestricted re-use,

distribution, and reproduction in any medium, provided the original work is properly cited.

\title{
Completeness of Infinite-dimensional Lie Groups in Their Left Uniformity
}

\author{
Helge Glöckner
}

\begin{abstract}
We prove completeness for the main examples of infinite-dimensional Lie groups and some related topological groups. Consider a sequence $G_{1} \subseteq G_{2} \subseteq \cdots$ of topological groups $G_{n}$ such that $G_{n}$ is a subgroup of $G_{n+1}$ and the latter induces the given topology on $G_{n}$, for each $n \in \mathbb{N}$. Let $G$ be the direct limit of the sequence in the category of topological groups. We show that $G$ induces the given topology on each $G_{n}$ whenever $\cup_{n \in \mathbb{N}} V_{1} V_{2} \cdots V_{n}$ is an identity neighbourhood in $G$ for all identity neighbourhoods $V_{n} \subseteq G_{n}$. If, moreover, each $G_{n}$ is complete, then $G$ is complete. We also show that the weak direct product $\oplus_{j \in J} G_{j}$ is complete for each family $\left(G_{j}\right)_{j \in J}$ of complete Lie groups $G_{j}$. As a consequence, every strict direct limit $G=\cup_{n \in \mathbb{N}} G_{n}$ of finite-dimensional Lie groups is complete, as well as the diffeomorphism group $\operatorname{Diff}_{c}(M)$ of a paracompact finite-dimensional smooth manifold $M$ and the test function group $C_{c}^{k}(M, H)$, for each $k \in \mathbb{N}_{0} \cup\{\infty\}$ and complete Lie group $H$ modelled on a complete locally convex space.
\end{abstract}

\section{Introduction and Statement of the Main Results}

Our main goal is to study completeness for Lie groups modelled on locally convex spaces (in the sense of [16,36]; $c f$. also [24,33,34]), and more generally completeness of topological groups, as far as this is useful for the main goal. Here completeness refers to the left uniform structure [25]. The topological groups under consideration need not be Hausdorff (unless we say so explicitly). It is well known that every Lie group $G$ modelled on a Banach space $E$ is complete [10, Chapter III, §1.1, Proposition 1]), as the left uniform structure and the one induced by the additive group of the Banach space coincide on some identity neighbourhood $U \subseteq G$ that is homeomorphic to a closed 0 -neighbourhood $V \subseteq E$.

Projective limits of complete Hausdorff groups being complete, this implies that many Fréchet-Lie groups are complete, e.g., the mapping groups

$$
C^{\infty}(M, H)=\lim _{\longleftarrow} C^{k}(M, H)
$$

that are the projective limit of the Banach-Lie groups $C^{k}(M, H)$ for $k \in \mathbb{N}_{0}$, for each compact smooth manifold $M$ and Banach-Lie group $H$. As usual, Lie groups modelled on Fréchet spaces (resp., Banach spaces) are called Fréchet-Lie groups (resp., Banach-Lie groups) in the following. Of course, completeness properties of locally convex spaces $E$, which furnish examples of abelian Lie groups $(E,+)$, are a standard

Received by the editors June 25, 2017; revised October 18, 2017.

Published electronically April 27, 2018.

AMS subject classification: 22E65, 22A05, 22E67, 46A13, 46M40, 58D05.

Keywords: infinite-dimensional Lie group, direct sum, box product, weak direct product, (LB)space, inductive limit, direct limit, ascending sequence, product set, bamboo shoot topology, compact support, test function group, diffeomorphism group, Banach-Lie group, left uniform structure, Cauchy net, Cauchy filter, strong (ILB)-Lie group, projective limit, inverse limit. 
topic in functional analysis. Moreover, while completeness properties of topological groups have been studied (see $[2,40]$ and the references therein), no systematic study of completeness properties of infinite-dimensional Lie groups is available so far. For example, it is an open question whether completeness of the modelling space implies completeness for a Lie group [36, Problem II.9]. The current article strives to develop specific tools that enable completeness to be shown for important classes of infinitedimensional Lie groups, under natural hypotheses. Our main results, Theorems 1.1 and 1.2, are devoted to completeness properties of direct limits. Recall that an ascending sequence $G_{1} \subseteq G_{2} \subseteq \cdots$ of topological groups $\left(G_{n}, \mathcal{O}_{n}\right)$ is a direct sequence of topological groups if, for each $n \in \mathbb{N}$, the inclusion map $j_{n+1, n}:\left(G_{n}, \mathcal{O}_{n}\right) \rightarrow\left(G_{n+1}, \mathcal{O}_{n+1}\right)$ is a continuous homomorphism. If, moreover, each $j_{n+1, n}$ is a topological embedding, i.e., a homeomorphism onto its image, then the direct sequence is called strict. Give $G:=\bigcup_{n \in \mathbb{N}} G_{n}$ the unique group structure for which each inclusion map $j_{n}: G_{n} \rightarrow G$ is a group homomorphism. A topology on a group is called a group topology if it makes it a topological group. There is a finest group topology $\mathcal{O}_{T G}$ on $G$ making each $j_{n}$ continuous; $\left(G, \mathcal{O}_{T G}\right)$ is called the direct limit topological group. The topology $\mathcal{O}_{T G}$ must not be confused with the final topology $\mathcal{O}_{D L}$ on $G$ with respect to the inclusion maps $j_{n}$, which makes $\left(G, \mathcal{O}_{D L}\right)$ the direct limit topological space. It is clear that $\mathcal{O}_{T G} \subseteq \mathcal{O}_{D L}$, but examples show that equality need not hold $[42,46]$.

Let $\mathcal{O}$ be a group topology on $G$ making each $j_{n}$ continuous. Following [21], we say that product sets are large in $(G, \mathcal{O})$ if

$$
\bigcup_{n \in \mathbb{N}} V_{1} V_{2} \cdots V_{n}
$$

is an identity neighbourhood in $(G, \mathcal{O})$ for every identity neighbourhood $V_{n}$ in $\left(G_{n}, \mathcal{O}_{n}\right) .{ }^{1}$ If product sets are large in $(G, \mathcal{O})$, then $\mathcal{O}=\mathcal{O}_{T G}$ [21, Proposition 11.8], and moreover the product sets as in (1.1) form a basis of identity neighbourhoods for $(G, \mathcal{O})$ (as we will note in Lemma 4.2). We mention that sets of the form

$$
\bigcup_{n \in \mathbb{N}}\left(V_{n} \cdots V_{2} V_{1}\right)\left(V_{1} V_{2} \cdots V_{n}\right)
$$

(so-called "bamboo shoots") were already used $[26,42]$ to obtain tangible descriptions of the topology $\mathcal{O}_{T G}$ in well-behaved situations. Our main result can be formulated as follows.

Theorem 1.1 Let $G_{1} \subseteq G_{2} \subseteq \cdots$ be a strict direct sequence of topological groups and $G:=\cup_{n \in \mathbb{N}} G_{n}$ be its direct limit topological group.

(i) If product sets are large in $G$, then each inclusion map $G_{n} \rightarrow G$ is a topological embedding.

(ii) If product sets are large in $G$ and each $G_{n}$ is complete, then $G$ is also complete.

We mention that $\left(G, \mathcal{O}_{T G}\right)$ is Hausdorff if each $G_{n}$ is Hausdorff and the inclusion map $G_{n} \rightarrow G$ is a topological embedding. ${ }^{2}$

\footnotetext{
${ }^{1}$ In [3], $(G, \mathcal{O})$ is then said to carry the strong topology.

${ }^{2}$ If $e \neq x \in G_{n}$, there is an open identity neighbourhood $V \subseteq G_{n}$ with $x \notin V$. Let $W \subseteq G$ be an open identity neighbourhood such that $W \cap G_{n}=V$. Then $x \notin W$. Hence $\overline{\{e\}}=\{e\}$ in $G$ and thus $G$ is Hausdorff.
} 
Theorem 1.1 and its proof were inspired by Bourbaki's discussion of completeness for strict direct limits of complete locally convex spaces [9, Chapter II, $\$ 4$, no. 6 , Proposition 9].

Now consider a family $\left(G_{j}\right)_{j \in J}$ of Lie groups $G_{j}$ modelled on locally convex spaces $E_{j}$. Then the so-called weak direct product

$$
G:=\bigoplus_{j \in J} G_{j}:=\left\{\left(x_{j}\right)_{j \in J} \in \prod_{j \in J} G_{j}: x_{j}=e \text { for almost all } j\right\}
$$

can be made a Lie group modelled on the locally convex direct sum $E:=\bigoplus_{j \in J} E_{j}$ in such a way that for some $C^{\infty}$-diffeomorphisms $\phi_{j}: U_{j} \rightarrow V_{j}$ from an open identity neighbourhood $U_{j} \subseteq G_{j}$ onto an open 0-neighbourhood $V_{j} \subseteq E_{j}$ with $\phi_{j}(e)=0$, the set $\bigoplus_{j \in J} U_{j}:=G \cap \prod_{j \in J} U_{j}$ is an open identity neighbourhood in $G$ and the map

$$
\bigoplus_{j \in J} \phi_{j}: \bigoplus_{j \in J} U_{j} \longrightarrow \bigoplus_{j \in J} V_{j} \subseteq E, \quad\left(x_{j}\right)_{j \in J} \longmapsto\left(\phi_{j}\left(x_{j}\right)\right)_{j \in J}
$$

is a $C^{\infty}$-diffeomorphism (as in [18]). If $J$ is countable, then the topological group underlying the weak direct product $\oplus_{j \in J} G_{j}$ is the small box product of the topological groups $G_{j}$ (as in [4]). If $J$ is uncountable, then the weak direct product and the small box product still coincide as groups, but the box topology is coarser and can be properly coarser. For example, this happens for the family $(\mathbb{R})_{j \in J}$ for an uncountable set $J$. The weak direct product $\mathbb{R}^{(J)}:=\bigoplus_{j \in J} \mathbb{R}$ then coincides with the locally convex direct sum, whose topology differs from the box topology, ${ }^{3}$ as is well known [43].

Theorem 1.2 Let $\left(G_{j}\right)_{j \in J}$ be a family of Lie groups $G_{j}$ modelled on locally convex spaces. If each $G_{j}$ is complete (resp., sequentially complete), then also the weak direct product $\oplus_{j \in J} G_{j}$ is complete (resp., sequentially complete).

Similarly, one finds that the small box product of each family of complete (resp., sequentially complete) topological groups is complete (resp., sequentially complete), see Example 5.2.

We now explain how the main results (and further findings) can be used to establish completeness for infinite-dimensional Lie groups within the main classes of examples [36, pp. 3-4], and related topological groups.

\subsection{Direct Limits of Finite-dimensional Lie Groups}

If $G_{1} \subseteq G_{2} \subseteq \cdots$ is a direct sequence of topological groups and the direct limit topology $\mathcal{O}_{D L}$ on $G=\bigcup_{n \in \mathbb{N}} G_{n}$ makes $G$ a topological group, i.e., if $\mathcal{O}_{T G}=\mathcal{O}_{D L}$, then product sets are large in $\left(G, \mathcal{O}_{T G}\right)[17$, Proposition 11.3]. Thus Theorem 1.1 entails the following corollary.

Corollary 1.3 If $\mathcal{O}_{T G}=\mathcal{O}_{D L}$ on $G=\bigcup_{n \in \mathbb{N}} G_{n}$ for a strict direct sequence $G_{1} \subseteq G_{2} \subseteq \cdots$ of complete topological groups, then $\left(G, \mathcal{O}_{T G}\right)$ is complete.

\footnotetext{
${ }^{3}$ In fact, $\left\{\left(x_{j}\right)_{r \in J} \in \mathbb{R}^{(J)}: \sum_{j \in J}\left|x_{j}\right|<1\right\}$ is a 0 -neighbourhood in the locally convex direct sum which cannot contain any box $\left.\bigoplus_{j \in J}\right]-q_{j}, q_{j}\left[\right.$ with $\left.q_{j} \in\right] 0, \infty\left[\cap \mathbb{Q}=: C\right.$ as one of the sets $J_{q}:=\left\{j \in J: q_{j}=q\right\}$ with $q \in C$ must be uncountable and hence infinite.
} 
We mention that $\mathcal{O}_{T G}=\mathcal{O}_{D L}$ on $G=\bigcup_{n \in \mathbb{N}} G_{n}$ for a direct sequence $G_{1} \subseteq G_{2} \subseteq \cdots$ of locally compact Hausdorff topological groups [26, 42]. Hence every strict direct limit $G=\bigcup_{n \in \mathbb{N}} G_{n}$ of locally compact Hausdorff topological groups $G_{1} \subseteq G_{2} \subseteq \cdots$ is complete. In particular, the Lie groups $\lim G_{n}$ [19] are complete for each strict direct sequence $G_{1} \subseteq G_{2} \subseteq \cdots$ of finite-dimensional Lie groups. ${ }^{4}$

\subsection{Diffeomorphism Groups}

For $M$ a paracompact finite-dimensional smooth manifold, consider the group $\operatorname{Diff}_{c}(M)$ of all $C^{\infty}$-diffeomorphisms $\phi: M \rightarrow M$ with compact support (in the sense that $\phi(x)=x$ for $x$ outside some compact set). Then $\operatorname{Diff}_{c}(M)$ is a Lie group modelled on the space of smooth compactly supported vector fields on $M$ (and $\operatorname{Diff}(M)$ can be made a Lie group with $\operatorname{Diff}_{c}(M)$ as an open normal subgroup) [33]. For each compact subset $K \subseteq M$,

$$
\operatorname{Diff}_{K}(M):=\left\{\phi \in \operatorname{Diff}_{c}(M):(\forall x \in M \backslash K) \phi(x)=x\right\}
$$

is a Lie subgroup of $\operatorname{Diff}_{c}(M)$, modelled on the Fréchet space of all smooth vector fields supported in $K$. If $M$ is $\sigma$-compact and $K_{1} \subseteq K_{2} \subseteq \cdots$ an exhaustion of $M$ by compact sets, ${ }^{5}$ then

$$
\operatorname{Diff}_{K_{1}}(M) \subseteq \operatorname{Diff}_{K_{2}}(M) \subseteq \cdots
$$

is a strict direct sequence of Lie groups. By [21, Example 11.7], the map

$$
\pi: \bigoplus_{n \in \mathbb{N}} \operatorname{Diff}_{K_{n}}(M) \longrightarrow \operatorname{Diff}_{c}(M)
$$

taking $\left(\phi_{1}, \ldots, \phi_{n}, \operatorname{id}_{M}, \operatorname{id}_{M}, \ldots\right)$ to $\phi_{1} \circ \ldots \circ \phi_{n}$ admits a smooth local section around $\operatorname{id}_{M}$ (in the spirit of fragmentation techniques familiar in the theory of diffeomorphism groups [6]. By [21, Remark 11.5, Proposition 11.8], this implies that product sets are large in $\operatorname{Diff}_{c}(M)$ and $\operatorname{Diff}_{c}(M)$ is the direct limit topological group of (1.2)), as recorded in [21, Proposition 5.4] (see [5, Remark 1, Proposition 1] for these arguments). ${ }^{6}$

Now Diff $K_{n}(M)$ is a strong (ILB)-Lie group (as considered in [38]) for each $n \epsilon$ $\mathbb{N}$. Using that strong (ILB)-Lie groups are complete (see Proposition 6.1 and Remark 6.2 (a)), Theorem 1.1 implies that $\operatorname{Diff}_{c}(M)$ is complete for $\sigma$-compact $M$ (see Remark 6.2 (b) and (c) for details).

If $M$ is merely paracompact and $\left(M_{j}\right)_{j \in J}$ its family of connected components, then $\operatorname{Diff}_{c}(M)$ has an open subgroup $G$ that is isomorphic to the weak direct product $\oplus_{j \in J} \operatorname{Diff}_{c}\left(M_{j}\right)$ as a Lie group, and we deduce with Theorem 1.2 that $G$ (and hence $\left.\operatorname{Diff}_{c}(M)\right)$ is complete also for paracompact $M$.

The completeness of diffeomorphism groups contrasts the incompleteness of many groups of homeomorphisms, among which one finds typical examples of metrizable topological groups that cannot be completed, as the sets of Cauchy sequences for the left and right uniformity do not coincide [15].

\footnotetext{
${ }^{4}$ The Lie group structure on important examples of such groups (like $\mathrm{GL}_{\infty}(\mathbb{R})=\underline{\lim _{\mathrm{GL}}} \mathrm{GL}_{n}(\mathbb{R})$ and $\left.\mathrm{SL}_{\infty}(\mathbb{R})=\lim _{\longrightarrow} \mathrm{SL}_{n}(\mathbb{R})\right)$ was already constructed $[32,35]$.

${ }^{5}$ Thus $\overrightarrow{M=} \cup_{n \in \mathbb{N}} K_{n}$ and $K_{n} \subseteq K_{n+1}^{0}$ for each $n \in \mathbb{N}$.

${ }^{6}$ We mention that the topology on the Lie group $\operatorname{Diff}_{c}(M)$ coincides with the Whitney $C^{\infty}$-topology used in [5]; this is clear from the description of this topology in [30] (see also [28] for a detailed account).
} 
Completeness of Infinite-dimensional Lie Groups in Their Left Uniformity

\subsection{Mapping Groups and Gauge Groups}

Among the prime examples of infinite-dimensional Lie groups are the Lie groups $C^{k}(M, H)$ of $C^{k}$-maps from a compact manifold $M$ to a Lie group $H$ for $k \in \mathbb{N}_{0} \cup\{\infty\}$ (notably the loop groups with $M=\mathbb{S}^{1}$ the circle group [39]), see [34,38]. More generally, if $M$ is a paracompact finite-dimensional smooth manifold and $H$ a Lie group modelled on a locally convex space $E$, there is a natural Lie group structure on the group $C_{c}^{k}(M, H)$ of all $C^{k}$-maps $\gamma: M \rightarrow H$ whose support

$$
\operatorname{supp}(\gamma):=\overline{\{x \in M: \gamma(x) \neq e\}}
$$

is compact (where $e$ is the neutral element of $H$ ), which is modelled on the locally convex space $C_{c}^{k}(M, E)$; see $[1,17]$ if $M$ is $\sigma$-compact. In the general case, we let $\left(M_{j}\right)_{j \in J}$ be the family of connected components of $M$ and use the group isomorphism

$$
C_{c}^{k}(M, H) \rightarrow \bigoplus_{j \in J} C_{c}^{k}\left(M_{j}, H\right), \quad \gamma \mapsto\left(\left.\gamma\right|_{M_{j}}\right)_{j \in j}
$$

to transport the Lie group structure of the weak direct product to the left-hand side. Using Theorems 1.1 and 1.2, we shall see that $C_{c}^{k}(M, H)$ is complete whenever $H$ and its modelling space $E$ are complete (Proposition 7.5). Likewise, gauge groups and full symmetry groups of principal bundles $[41,45]$ are complete if the structure group $H$ and its modelling space are complete (see Remark 7.6 for more details).

\subsection{Linear Lie Groups}

We can also prove completeness for some unit groups of topological algebras. ${ }^{7}$ Consider an ascending sequence $A_{1} \subseteq A_{2} \subseteq \cdots$ of unital Banach algebras, such that all inclusion maps $A_{n} \rightarrow A_{n+1}$ are continuous homomorphisms of unital algebras. Endow $A:=\bigcup_{n \in \mathbb{N}} A_{n}$ with the unital algebra structure turning each inclusion map $A_{n} \rightarrow A$ into a homomorphism of unital algebras. Then the locally convex direct limit topology makes $A$ a topological algebra and product sets are large in $A^{\times}=\bigcup_{n \in \mathbb{N}} A_{n}^{\times}$[21, Proposition 12.1 (a), (c) ], [14]. With Theorem 1.1, we deduce that $A^{\times}$(like each $A_{n}^{\times}$) is complete whenever the direct sequence $A_{1} \subseteq A_{2} \subseteq \cdots$ is strict.

\subsection{Ascending Unions of Banach-Lie Groups}

Beyond unit groups of Banach algebras, let us consider an ascending sequence $G_{1} \subseteq$ $G_{2} \subseteq \cdots$ of Banach-Lie groups over $\mathbb{K} \in\{\mathbb{R}, \mathbb{C}\}$ such that each inclusion map $G_{n} \rightarrow G_{n+1}$ is a $\mathbb{K}$-analytic group homomorphism. In [12, Theorem C], conditions were spelled out that ensure that $G=\bigcup_{n \in \mathbb{N}} G_{n}$ can be made a $\mathbb{K}$-analytic Lie group modelled on the locally convex direct limit of the respective Lie algebras. We show that product sets are large in the Lie groups $G$ constructed in [12, Theorem $C]$, whence the given topology on $G$ makes it the direct limit topological group $G=\lim _{\longrightarrow} G_{n}$ (Proposition 8.2). Using Theorem 1.1, we deduce that $G=\cup_{n \in \mathbb{N}} G_{n}$ (as before) is complete whenever the direct sequence $G_{1} \subseteq G_{2} \subseteq \cdots$ is strict (Proposition 8.2).

For a concrete example, let $\left(F_{n},\|\cdot\|_{n}\right)_{n \in \mathbb{N}}$ be a sequence of Banach spaces. Write $\mathcal{L}(F)$ for the Banach algebra of bounded operators $S: F \rightarrow F$ for a Banach space $F$,

\footnotetext{
${ }^{7}$ If $A$ is a unital algebra, we write $A^{\times}$for its unit group of all invertible elements.
} 
endowed with the operator norm $\|\cdot\|_{\text {op }}$. We equip $E_{n}:=F_{1} \oplus \cdots \oplus F_{n}$ with the maximum norm and identify GL $\left(E_{n}\right):=\mathcal{L}\left(E_{n}\right)^{\times}$with the subgroup $\mathrm{GL}\left(E_{n}\right) \times\left\{\operatorname{id}_{F_{n+1}}\right\}$ of $\operatorname{GL}\left(E_{n+1}\right)$. Using [13, Theorem A], it was shown in [11, Theorem 38] that

$$
\mathrm{GL}\left(\left(F_{n}\right)_{n \in \mathbb{N}}\right):=\bigcup_{n \in \mathbb{N}} \mathrm{GL}\left(E_{n}\right)
$$

can be made into a Lie group. ${ }^{8}$ As the direct sequence $\mathrm{GL}\left(E_{1}\right) \subseteq \mathrm{GL}\left(E_{2}\right) \subseteq \cdots$ is strict, the preceding reasoning shows that $\mathrm{GL}\left(\left(F_{n}\right)_{n \in \mathbb{N}}\right)$ is complete.

\subsection{Outlook: Lie Groups Modelled on Silva Spaces}

Work by Hunt and Morris [29] implies that every Lie group modelled on a Silva space $^{9}$ is complete [22, Corollary 1.4]. This entails that direct limits $\cup_{n \in \mathbb{N}} G_{n}$ of finitedimensional Lie groups (or locally compact groups) $G_{1} \subseteq G_{2} \subseteq \cdots$ are always complete (no matter whether the direct sequence is strict or not). It also shows that the Lie group Diff ${ }^{\omega}(M)$ of real-analytic diffeomorphisms is complete for each compact realanalytic manifold $M$, as well as the Lie group $C^{\omega}(M, H)$ of all real-analytic $H$-valued mappings on the latter, for each finite-dimensional Lie group $H$ [22].

\section{Preliminaries and Notation}

We write $\mathbb{N}=\{1,2, \ldots\}$ and $\mathbb{N}_{0}:=\mathbb{N} \cup\{0\}$. Topological groups and locally convex (real topological vector) spaces shall not be assumed to be Hausdorff, unless we say so explicitly. If $f: X \rightarrow Y$ is a function between metric spaces $\left(X, d_{X}\right)$ and $\left(Y, d_{Y}\right)$, we define

$$
\operatorname{Lip}(f):=\sup \left\{\frac{d_{Y}(f(x), f(y))}{d_{X}(x, y)}: x \neq y \in X\right\} \in[0, \infty]
$$

and call $f$ Lipschitz if $\operatorname{Lip}(f)<\infty$. If each point $x \in X$ has a neighbourhood $V \subseteq X$ such that $\left.f\right|_{V}: V \rightarrow Y$ is Lipschitz (with respect to the metric $\left.d_{X}\right|_{V \times V}$ induced on $V$ ), then $f$ is called locally Lipschitz. If $(E,\|\cdot\|)$ is a Banach space, we write $\operatorname{GL}(E)$ for the group of continuous automorphisms of the vector space $E$. For $x \in E$ and $r>0$, we write $B_{r}^{E}(x):=\{y \in E:\|y-x\|<r\}$ and $\bar{B}_{r}^{E}(x):=\{y \in E:\|y-x\| \leq r\}$. If $q$ is a continuous seminorm on a locally convex space $E$, we write

$$
\bar{B}_{r}^{q}(0):=\{x \in E: q(x) \leq r\}
$$

for $r>0$.

Remark 2.1 For our setting of $C^{k}$-maps, real and complex analytic mappings between open subsets of locally convex Hausdorff topological vector spaces, the reader is referred to $[16,36]$, where the corresponding concepts of manifolds (and Lie groups) modelled on Hausdorff locally convex spaces are also described ( $c f$. also $[8,24,33$, 34]). ${ }^{10}$ All of these manifolds (and Lie groups) are Hausdorff. Every Lie group $G$

\footnotetext{
${ }^{8} \operatorname{In~[11],~GL}\left(\left(F_{n}\right)_{n \in \mathbb{N}}\right)$ is denoted by $\mathrm{GL}(E)$, with $E:=\bigcup_{n \in \mathbb{N}} E_{n}$.

${ }^{9} \mathrm{~A}$ locally convex space is called a Silva space or (DFS)-space if it is a locally convex direct $\operatorname{limit} \lim E_{n}$ for an ascending sequence $E_{1} \subseteq E_{2} \subseteq \cdots$ of Banach spaces, such that all inclusion maps $E_{n} \rightarrow E_{n+1}$ are compact operators.

${ }^{10}$ The $C^{k}$-maps are those of Keller's $C_{c}^{k}$-theory [31], going back to A. Bastiani [7].
} 
is a topological group in its given topology (as smooth maps are continuous in the infinite-dimensional calculus we are using). We write $e$ for the neutral element of $G$ and $L(G):=T_{e}(G)$ (or simply $\mathfrak{g}$ ) for its Lie algebra (the tangent space at $e$ ). We write $\exp _{G}: L(G) \rightarrow G$ for the exponential function of $G$, if it exists [34,36]. If $f: G \rightarrow H$ is a smooth group homomorphism between Lie groups, we abbreviate $L(f):=T_{e}(f)$. If $M$ is a $C^{k}$-manifold with $k \geq 1$, we let $\pi_{T M}: T M \rightarrow M$ be the bundle projection. If $E$ is a locally convex space and $U \subseteq E$ an open subset, we identify the tangent bundle $T U$ with $U \times E$ in the usual way. If $M$ is a $C^{1}$-manifold and $f: M \rightarrow U$ a $C^{1}$-map, we write $d f: T M \rightarrow E$ for the second component of $T f: T M \rightarrow T U=U \times E$. If $g: U \rightarrow F$ is a $C^{1}$-map to a locally convex space, we write $g^{\prime}(x):=d g(x, \cdot): E \rightarrow F$ for $x \in U$.

The following fact is based on the Mean Value Theorem.

Lemma 2.2 Let $\left(E,\|\cdot\|_{E}\right)$ and $\left(F,\|\cdot\|_{F}\right)$ be normed spaces, let $U \subseteq E$ be open and let $f: U \rightarrow F$ be a $C^{1}$-map. Then the following hold.

(i) $f$ is locally Lipschitz.

(ii) If $U$ is convex, then $f$ is Lipschitz if and only if $L:=\sup _{x \in U}\left\|f^{\prime}(x)\right\|_{o p}<\infty$, in which case $\operatorname{Lip}(f)=L$.

Recall that a net $\left(x_{\alpha}\right)_{\alpha \in A}$ in a topological group $G$ is called a left Cauchy net if, for each identity neighbourhood $U \subseteq G$, there exists $\gamma \in A$ such that $x_{\beta}^{-1} x_{\alpha} \in U$ for all $\alpha, \beta \geq \gamma$. If every left Cauchy net in $G$ is convergent, then $G$ is called complete; if every left Cauchy sequence in $G$ is convergent, then $G$ is sequentially complete.

\subsection{Facts Concerning Completeness}

Many results concerning completeness of topological groups can be found in [40]. We mention useful facts.

Proposition 2.3 (i) If a topological group $G$ is complete (resp., sequentially complete), then every closed subgroup $H \subseteq G$ is complete in the induced topology.

(ii) For every family $\left(G_{j}\right)_{j \in J}$ of topological groups that are complete (resp., sequentially complete), the direct product $\prod_{j \in J} G_{j}$ is complete (resp., sequentially complete) in the product topology.

(iii) Let $\left(\left(G_{j}\right)_{j \in J},\left(q_{i, j}\right)_{i \leq j}\right)$ be a projective system of Hausdorff topological groups $G_{j}$ and continuous homomorphisms $q_{i, j}: G_{j} \rightarrow G_{i}$ for $i \leq j$ in $J$ such that $q_{i, i}=\mathrm{id}_{G_{i}}$ and $q_{i, j} \circ q_{j, k}=q_{i, k}$ whenever $i \leq j \leq k$. If each $G_{i}$ is complete (resp., sequentially complete), then also the projective limit $\lim _{\longleftarrow} G_{j}$ is complete (resp., sequentially complete), as it can be realized as the closed subgroup

$$
\left\{\left(x_{j}\right)_{j \in J} \in \prod_{j \in J} G_{j}:(\forall i \leq j) x_{i}=q_{i, j}\left(x_{j}\right)\right\}
$$

of the direct product, endowed with the induced topology.

(iv) Completeness is an extension property: If $G$ is a topological group and $N \subseteq G$ a normal subgroup such that both $N$ and $G / N$ are complete, then $G$ is also complete [40, Theorem $12.3(\mathrm{a})]$. 
If $G$ is a topological group, $H \subseteq G$ a subgroup and $N \subseteq G$ a normal subgroup, we say that $G$ is the (internal) semidirect product of $N$ and $H$ as topological groups if the product map $N \times H \rightarrow G,(x, y) \mapsto x y$ is a homeomorphism. Since $q: G \rightarrow H, x y \mapsto y$ is a quotient homomorphism with kernel $N$, the following holds as special case of (iv):

(v) Let $G$ be a topological group which, as a topological group, is the internal semidirect product of a normal subgroup $N$ and a subgroup $H$. If $N$ and $H$ are complete, then $G$ is also complete [40, Proposition 12.5 (a)].

The following slight generalization of Proposition 2.3 (iii) is useful.

Lemma 2.4 Let $G$ be a topological group whose underlying topological space is the projective limit of a projective system $\left(\left(X_{j}\right)_{j \in J},\left(q_{i, j}\right)_{i \leq j}\right)$ of Hausdorff topological spaces $X_{j}$, with limit maps $q_{j}: G \rightarrow X_{j}$ for $j \in J$. Assume that for each Cauchy net $\left(x_{\alpha}\right)_{\alpha \in A}$ in $G$, the corresponding net $\left(q_{j}\left(x_{\alpha}\right)\right)_{\alpha \in A}$ converges in $X_{j}$, for each $j \in J$. Then $G$ is complete.

Proof We can assume that $G=\left\{\left(x_{j}\right)_{j \in J} \in \prod_{j \in J} X_{j}:(\forall i \leq j) x_{i}=q_{i, j}\left(x_{j}\right)\right\}$ and $q_{i}\left(\left(x_{j}\right)_{j \in J}\right)=x_{i}$ for all $i \in I$ and $x=\left(x_{j}\right)_{j \in J} \in G$. For $j \in J$, let $y_{j} \in X_{j}$ be the limit of $\left(q_{j}\left(x_{\alpha}\right)\right)_{\alpha \in A}$. For $i, j \in I$ with $i \leq j$, the net $\left(q_{i}\left(x_{\alpha}\right)\right)_{\alpha \in A}=\left(q_{i, j}\left(q_{j}\left(x_{\alpha}\right)\right)\right)_{\alpha \in A}$ in $X_{i}$ converges both to $y_{i}$ and $q_{i, j}\left(y_{j}\right)$. Since $X_{i}$ is Hausdorff, $y_{i}=q_{i, j}\left(y_{j}\right)$ follows. Hence $y:=\left(y_{j}\right)_{j \in J} \in G$ and as $q_{j}\left(x_{\alpha}\right) \rightarrow y_{j}=q_{j}(y)$ for all $j \in J$, the net $\left(x_{\alpha}\right)_{\alpha \in A}$ converges to $y$.

Lemma 2.5 ([4]) If $\left(G_{j}\right)_{j \in J}$ is a family of topological groups, let $\square_{j \in J} G_{j} \subseteq \prod_{j \in J} G_{j}$ be the subgroup of all $\left(x_{j}\right)_{j \in J} \in \prod_{j \in J} G_{j}$ such that $x_{j}=e$ for all but finitely many $j$. Consider the sets $\square_{j \in J} U_{j}:=\prod_{j \in J} U_{j} \cap \square_{j \in J} G_{j}$, for $\left(U_{j}\right)_{j \in J}$ ranging through the families of open subsets $U_{j} \subseteq G_{j}$ such that $e \in U_{j}$ for all but finitely many $j$. The latter sets form a basis for a topology on $\square_{j \in J} G_{j}$ making it a topological group called the box topology. When endowed with this topology, $\square,{ }_{j \in J} G_{j}$ is called the small box product of the family $\left(G_{j}\right)_{j \in J}$.

If each $G_{j}$ is a Lie group modelled on a Hausdorff locally convex space then, $\square_{j \in J} G_{j}$ is also a Lie group in a natural way. (See [23], where small box products are called weak direct products, in contrast to the conventions here.) It is modelled on the small box product $\square{ }_{j \in J} E_{j}$. If, instead, we use the locally convex direct sum as the modelling space, then the group $\square_{j \in J} G_{j}$ can also be made into a Lie group, called the weak direct product of the family and denoted here by $\oplus_{j \in J} G_{j}$ (see [18], where the notation $\Pi_{j \in J}^{*} G_{j}$ is used). The two possible modelling spaces (and the two Lie groups) coincide if $J$ is countable. When dealing with $\oplus_{j \in J} G_{j}$, we write $\oplus_{j \in J} U_{j}$, instead of $\square_{j \in J} U_{j}$.

\section{Exploiting Completeness of a Subgroup}

The following lemma is essential for the proof of Theorem 1.1.

Lemma 3.1 Let $\left(x_{\alpha}\right)_{\alpha \in A}$ be a left Cauchy net in a topological group $G$ and $H \subseteq G$ be a subgroup that is a complete topological group in the induced topology. Assume that, for 
each $\alpha \in A$ and identity neighbourhood $W \subseteq G$, there exists $\beta \geq \alpha$ such that $x_{\beta} \in H W$. Then $\left(x_{\alpha}\right)_{\alpha \in A}$ converges in $G$ to some $y \in H$.

Proof Let $U$ be the set of all identity neighborhoods in $G$. By hypothesis,

$$
A_{W}:=\left\{\alpha \in A: x_{\alpha} \in H W\right\}
$$

is cofinal in $A$ for all $W \in \mathcal{U}$ and thus $M:=\left\{(W, \alpha) \in \mathcal{U} \times A: \alpha \in A_{W}\right\}$ becomes a directed set if we write $\left(W_{1}, \alpha_{1}\right) \leq\left(W_{2}, \alpha_{2}\right)$ if and only if $W_{2} \subseteq W_{1}$ and $\alpha_{1} \leq \alpha_{2}$. For $a=(W, \alpha) \in M$, pick $y_{a} \in H$ and $w_{a} \in W$ such that

$$
x_{\alpha}=y_{a} w_{a} .
$$

Then $\left(y_{a}\right)_{a \in M}$ is a left Cauchy net in $H$. In fact, if $U$ is an identity neighborhood in $H$, we find $Q \in \mathcal{U}$ such that $U=H \cap Q$. Let $P \in \mathcal{U}$ such that $P P P^{-1} \subseteq Q$ and $\gamma \in A$ such that $x_{\beta}^{-1} x_{\alpha} \in P$ for all $\alpha, \beta \geq \gamma$. We may assume that $\gamma \in A_{P}$. For all $a, b \geq(P, \gamma)$ in $M$, say $a=(W, \alpha)$ and $b=(V, \beta)$, we have $V, W \subseteq P$ and hence

$$
y_{b}^{-1} y_{a}=w_{b} x_{\beta}^{-1} x_{\alpha} w_{a}^{-1} \in V P W^{-1} \subseteq P P P^{-1} \subseteq Q .
$$

Thus $y_{b}^{-1} y_{a} \in H \cap Q=U$.

Let $y$ be the limit of $\left(y_{a}\right)_{a \in M}$ in $H$. Then $y_{a} \rightarrow y$ also in G. Given $W \in \mathcal{U}$, let $\alpha \in A_{W}$. Since $w_{a} \in W$ for $a \geq(W, \alpha)$, the net $\left(w_{a}\right)_{a \in M}$ converges to $e$ in $G$. Using (3.1), we deduce that the subnet $\left(x_{\alpha}\right)_{(W, \alpha) \in M}$ of $\left(x_{\alpha}\right)_{\alpha \in A}$, and hence also the Cauchy net $\left(x_{\alpha}\right)_{\alpha \in A}$, converges to $y$.

\section{Completeness of Strict Direct Limits}

In this section, we prove Theorem 1.1.

Lemma 4.1 Let $G$ be a group, $2 \leq n \in \mathbb{N}$, and $G_{1} \subseteq G_{2} \subseteq \cdots \subseteq G_{n}=G$ be subgroups. For $j \in\{1, \ldots, n\}$, let $W_{j}$ be a subset of $G_{j}$. Then

$$
G_{1} \cap\left(W_{1} W_{2} \cdots W_{n}\right)=G_{1} \cap\left(W_{1} \cdots W_{n-1}\left(G_{n-1} \cap W_{n}\right)\right) .
$$

Proof We show by induction on $n$ that the left-hand side of (4.1) is a subset of the right-hand side (the other inclusion is trivial), for all $G, G_{1}, \ldots, G_{n}$, and $W_{1}, \ldots, W_{n}$ as described in the lemma. If $n=2$ and $x \in G_{1} \cap W_{1} W_{2}$, then $x=w_{1} w_{2}$ with $w_{1} \in W_{1}$ and $w_{2} \in W_{2}$. Since $W_{1} \subseteq G_{1}$, we have $w_{1} \in G_{1}$ and thus $w_{2}=w_{1}^{-1} x \in G_{1} \cap W_{2}$.

If $n>2$ and the assertion holds for $n-1$, let $x \in G_{1} \cap\left(W_{1} \cdots W_{n}\right)$. Write $x=$ $w_{1} w_{2} \cdots w_{n}$ with $w_{j} \in W_{j}$ for $j \in\{1, \ldots, n\}$. Then

$$
w_{2} \cdots w_{n}=w_{1}^{-1} x \in G_{1} \cap\left(W_{2} \cdots W_{n}\right) \subseteq G_{2} \cap\left(W_{2} \cdots W_{n}\right)
$$

and thus $w_{2} \ldots w_{n} \in W_{2} \cdots W_{n-1}\left(G_{n-1} \cap W_{n}\right)$ by the inductive hypothesis.

Lemma 4.2 Assume that $G$ is the direct limit topological group of a direct sequence $G_{1} \subseteq G_{2} \subseteq \cdots$ of topological groups, and that product sets are large in $G$. Then every identity neighbourhood of $G$ contains a product set $\bigcup_{n \in \mathbb{N}} W_{1} W_{2} \cdots W_{n}$ for suitable identity neighbourhoods $W_{n} \subseteq G_{n}$. 
Proof If $V_{0}$ is an identity neighbourhood in $G$, there exist identity neighbourhoods $V_{n} \subseteq G$ for $n \in \mathbb{N}$ such that $V_{n} V_{n} \subseteq V_{n-1}$. Then $W_{n}:=G_{n} \cap V_{n}$ is an identity neighbourhood in $G_{n}$ and $V_{1} V_{2} \cdots V_{n} \subseteq V_{0}$ for all $n \in \mathbb{N}$ implies that

$$
\bigcup_{n \in \mathbb{N}} W_{1} \cdots W_{n} \subseteq \bigcup_{n \in \mathbb{N}} V_{1} \cdots V_{n} \subseteq V_{0} .
$$

Proof of Theorem 1.1 (i) To see that $G$ induces the given topology on $G_{n}$, we can assume that $n=1$. Let $V_{1} \subseteq G_{1}$ be an identity neighbourhood. There exists an identity neighbourhood $W_{1} \subseteq G_{1}$ such that $W_{1} W_{1} \subseteq V_{1}$. Recursively, for $m \geq 2$, find an identity neighbourhood $V_{m} \subseteq G_{m}$ such that $G_{m-1} \cap V_{m}=W_{m-1}$ (which is possible as $G_{m}$ induces the given topology on $\left.G_{m-1}\right)$ and an identity neighbourhood $W_{m} \subseteq G_{m}$ such that $W_{m} W_{m} \subseteq V_{m}$. Then $G_{1} \cap\left(W_{1} W_{2} \cdots W_{m}\right) \subseteq G_{1} \cap\left(W_{1} \cdots W_{j-1} V_{j}\right)$ for all $j \in$ $\{m, m-1, \ldots, 1\}$, by induction. If $j=m$, we have $W_{m} \subseteq V_{m}$ and the assertion holds. If $j \in\{2, \ldots, m\}$ and the assertion holds for $j$, then

$$
\begin{aligned}
& G_{1} \cap\left(W_{1} W_{2} \cdots W_{m}\right) \subseteq G_{1} \cap\left(W_{1} \cdots W_{j-1} V_{j}\right)=G_{1} \cap\left(W_{1} \cdots W_{j-1}\left(G_{j-1} \cap V_{j}\right)\right) \\
&=G_{1} \cap\left(W_{1} \cdots W_{j-1} W_{j-1}\right) \subseteq G_{1} \cap\left(W_{1} \cdots W_{j-2} V_{j-1}\right),
\end{aligned}
$$

using the inductive hypothesis, Lemma 4.1, the identity $G_{j-1} \cap V_{j}=W_{j-1}$, and the inclusion $W_{j-1} W_{j-1} \subseteq V_{j-1}$. Taking $j=1$, we deduce that $G_{1} \cap\left(W_{1} W_{2} \cdots W_{m}\right) \subseteq V_{1}$, for all $m \in \mathbb{N}$ and hence $G_{1} \cap W \subseteq V_{1}$ if we define $W:=\bigcup_{m \in \mathbb{N}} W_{1} W_{2} \cdots W_{m}$. As we assume that product sets are large in $G$, the set $W$ is an identity neighborhood in $G$. Since $G_{1} \cap W \subseteq V_{1}$, the group topology $\mathcal{T}$ induced by $G$ on $G_{1}$ is finer than the given topology $\mathcal{O}_{1}$ on $G_{1}$ and hence coincides with it (noting that $\mathcal{T} \subseteq \mathcal{O}_{1}$ as the inclusion map $\left(G_{1}, \mathcal{O}_{1}\right) \rightarrow G$ is continuous).

(ii) If each $G_{n}$ is complete, let $\left(x_{\alpha}\right)_{\alpha \in A}$ be a left Cauchy net in $G$. Let $\mathcal{U}$ be the set of all identity neighborhoods in $G$. We claim that there exists $m \in \mathbb{N}$ such that, for each $\alpha \in A$ and $W \in \mathcal{U}$, there exists $\beta \geq \alpha$ such that $x_{\beta} \in G_{m} W$. If this is true, then $\left(x_{\alpha}\right)_{\alpha \in A}$ converges in $G$ by Lemma 3.1 using that $G$ induces the given complete group topology on $G_{m}$, by (i).

To prove the claim, suppose it is wrong. Then, for each $m \in \mathbb{N}$, there exist $\alpha_{m} \in A$ and $W_{m} \in \mathcal{U}$ such that

$$
x_{\alpha} \notin G_{m} W_{m} \quad \text { for all } \alpha \geq \alpha_{m} .
$$

After shrinking $W_{m}$ if necessary, we can assume that $W_{m}=\bigcup_{n \in \mathbb{N}} W_{1}^{(m)} \cdots W_{n}^{(m)}$ with identity neighbourhoods $W_{n}^{(m)} \subseteq G_{n}$, by Lemma 4.2. After shrinking $W_{n}^{(2)}, W_{n}^{(3)}, \ldots$, we can assume that

$$
W_{n}^{(m+1)} \subseteq W_{n}^{(m)} \quad \text { for all } n, m \in \mathbb{N} .
$$

Since product sets are large in $G$, the set $W:=\bigcup_{n \in \mathbb{N}} W_{1}^{(1)} \cdots W_{n}^{(n)}$ is an identity neighbourhood in $G$. By (4.3), we have

$$
\bigcup_{n>m} W_{m+1}^{(m+1)} \cdots W_{n}^{(n)} \subseteq \bigcup_{n>m} W_{m+1}^{(m)} \cdots W_{n}^{(m)} \subseteq \bigcup_{n \in \mathbb{N}} W_{1}^{(m)} \cdots W_{n}^{(m)}=W_{m} .
$$

Using that $W_{1}^{(1)} \cdots W_{n}^{(n)} \subseteq G_{m}$ for $n \in\{1, \ldots, m\}$, we deduce that

$$
G_{m} W=G_{m} \bigcup_{n>m} W_{m+1}^{(m+1)} \cdots W_{n}^{(n)} \subseteq G_{m} W_{m}
$$


and thus

$$
G_{m} W \subseteq G_{m} W_{m} \quad \text { for all } m \in \mathbb{N} .
$$

By definition of a Cauchy net, we find $\gamma \in A$ such that

$$
x_{\alpha}^{-1} x_{\beta} \in W
$$

for all $\alpha, \beta \geq \gamma$. Now $x_{\gamma} \in G_{m_{0}}$ for some $m_{0} \in \mathbb{N}$. Since $A$ is directed, we find $\alpha \in A$ such that $\alpha \geq \gamma$ and $\alpha \geq \alpha_{m_{0}}$. Using (4.5), we obtain $x_{\alpha}=x_{\gamma}\left(x_{\gamma}^{-1} x_{\alpha}\right) \in G_{m_{0}} W$. But $x_{\alpha} \notin G_{m_{0}} W_{m_{0}}$ by (4.2) and thus $x_{\alpha} \notin G_{m_{0}} W$ by (4.4), which is absurd.

\section{Completeness of Weak Direct Products}

The following lemma will enable us to reduce the completeness of weak direct products (and box products) to that of direct products.

Lemma 5.1 Let $P$ be a complete, respectively, sequentially complete, topological group and $G$ be a subgroup of $P$, endowed with a topology $\mathcal{O}$ that is finer than the topology $\mathcal{T}$ induced by $P$ on $G$. Assume that, for each $x \in P$ such that $x \notin G$, there exists a closed subset $L$ in $P$ such that

(i) $G \cap L$ is an identity neighborhood in $(G, \mathcal{O})$,

(ii) $G \cap x L=\varnothing$.

Moreover, assume that

(iii) The closures $\bar{V}$ in $(G, \mathcal{T})$ form a basis of identity neighbourhoods in $(G, \mathcal{O})$, for $V$ in the set of identity neighbourhoods in $(G, \mathcal{O})$.

Then also $(G, \mathcal{O})$ is complete, respectively, sequentially complete.

Proof Assume that $P$ is complete. If $\left(x_{\alpha}\right)_{\alpha \in A}$ is a left Cauchy net in $(G, \mathcal{O})$, then it also is a left Cauchy net in $P$, whence $x_{\alpha} \rightarrow x$ in $P$ for some $x \in P$. Then $x \in G$, since otherwise we obtain a contradiction: let $L \subseteq P$ be a closed subset such that $G \cap L$ is an identity neighbourhood in $(G, \mathcal{O})$ and $G \cap x L=\varnothing$. Let $\alpha_{0} \in A$ such that $x_{\alpha}^{-1} x_{\beta} \in G \cap L$ for all $\alpha, \beta \geq \alpha_{0}$. Considering $x_{\alpha}^{-1} x_{\beta}$ as elements of $P$ and passing to the limit in $\alpha$, we obtain $x^{-1} x_{\beta} \in L$ for all $\beta \geq \alpha_{0}$, whence $x_{\beta} \in G \cap x L=\varnothing$, which is absurd.

Let $W$ be an identity neighborhood in $(G, \mathcal{O})$. By hypothesis (iii), we find an identity neighbourhood $V$ in $(G, \mathcal{O})$ such that $G \cap \bar{V} \subseteq W$, where $\bar{V}$ is the closure of $V$ in $P$. There exists $\alpha_{0} \in A$ such that $x_{\alpha}^{-1} x_{\beta} \in V$ for all $\alpha, \beta \geq \alpha_{0}$. Considering $x_{\alpha}^{-1} x_{\beta}$ as an element of $P$ and passing to the limit in $\alpha$, we deduce that $x^{-1} x_{\beta} \in \bar{V}$ for all $\beta \geq \alpha_{0}$, whence $x^{-1} x_{\beta} \in G \cap \bar{V} \subseteq W$ and thus $x_{\beta} \in x W$. Thus $x_{\beta} \rightarrow x$ in $(G, \mathcal{O})$.

If $P$ is sequentially complete, then the proof is identical with $A:=\mathbb{N}$.

Example 5.2 Let $\left(G_{j}\right)_{j \in J}$ be a family of topological groups $G_{j}$ that are complete, respectively, sequentially complete. Then also the small box product $G:=\square_{j \in J} G_{j}$ is complete, respectively, sequentially complete.

To see this, let us check that Lemma 5.1 can be used with $P:=\prod_{j \in J} G_{j}$. If

$$
x=\left(x_{j}\right)_{j \in J} \in P \backslash G,
$$


then $I:=\left\{j \in J: x_{j} \neq e\right\}$ is an infinite set. For each $j \in I$, let $U_{j} \subseteq G_{j}$ be a closed identity neigbourhood such that $x_{j}^{-1} \notin U_{j}$. For $j \in J \backslash I$, let $U_{j}:=G_{j}$. Then $L:=\prod_{j \in J} U_{j}$ is closed in $P$ and $G \cap L=\square_{j \in J} U_{j}$ is an identity neighbourhood such that $x L \cap G=\varnothing$ as each $y=\left(y_{j}\right)_{j \in J} \in x L$ satisfies $y_{j} \neq e$ for all $j \in I$. Finally, let $W \subseteq G$ be an identity neighbourhood. Then $W$ contains a box $V:=\square_{j \in J} V_{j}$ with closed identity neighbourhoods $V_{j} \subseteq G_{j}$. The closure $\bar{V}$ of $V$ in $P$ is $\prod_{j \in J} V_{j}$, whence $G \cap \bar{V}=V \subseteq W$.

Proof of Theorem 1.2 To verify the theorem, let us check that Lemma 5.1 can be applied with $P:=\prod_{j \in J} G_{j}$. If $x=\left(x_{j}\right)_{j \in J} \in P \backslash G$, then $I:=\left\{j \in J: x_{j} \neq e\right\}$ is an infinite set. For each $j \in I$, let $U_{j} \subseteq G_{j}$ be a closed identity neigbourhood such that $x_{j}^{-1} \notin U_{j}$. For $j \in J \backslash I$, let $U_{j}:=G_{j}$. Then $L:=\prod_{j \in J} U_{j}$ is closed in $P$ and $G \cap L=\square_{j \in J} U_{j}$ is an identity neighbourhood (as the topology on $G$ is finer than the box topology) such that $x L \cap G=\varnothing$. Thus $L$ satisfies the conditions (i) and (ii) in Lemma 5.1.

Next, let $S \subseteq G$ be an identity neighbourhood. For $j \in J$, let $E_{j}$ be the locally convex space on which $G_{j}$ is modelled. Then $S$ contains an identity neighbourhood of the form $W=\phi^{-1}(Q)$ for a diffeomorphism $\phi: U \rightarrow V$ and a 0-neighbourhood $Q \subseteq V$, where diffeomorphisms $\phi_{j}: U_{j} \rightarrow V_{j}$ from open identity neighbourhoods $U_{j} \subseteq G_{j}$ onto open 0-neighbourhoods $V_{j} \subseteq E_{j}$ are used to define $U:=\oplus_{j \in J} U_{j}$, $V:=\bigoplus_{j \in J} V_{j}$, and $\phi:=\bigoplus_{j \in J} \phi_{j}: U \rightarrow V$. For each $j \in J$, the topological group $G_{j}$ has a closed identity neighbourhood $K_{j}$ such that $K_{j} \subseteq U_{j}$. Set $L_{j}:=\phi_{j}\left(K_{j}\right)$. After shrinking $Q$ (and $W=\phi^{-1}(Q)$ ), we can assume that $W \subseteq \oplus_{j \in J} K_{j}=: K$ and thus $Q \subseteq \prod_{j \in J} L_{j}=: L$. After shrinking $Q$ further if necessary, we can also assume that $Q=\bar{B}_{r}^{q}(0)$ for a continuous seminorm $q$ on $\bigoplus_{j \in J} E_{j}$, and we may assume that $q$ is of the form $q(x)=\sum_{j \in J} q_{j}\left(x_{j}\right)$ for all $x=\left(x_{j}\right)_{j \in J} \in \bigoplus_{j \in J} E_{j}$, for certain continuous seminorms $q_{j}$ on $E_{j}$. Then the closure $\bar{Q}$ of $Q$ in $\prod_{j \in J} E_{j}$ is the set $C$ of all

$$
\left(x_{j}\right)_{j \in J} \in \prod_{j \in J} E_{j}
$$

such that $\sum_{j \in J} q_{j}\left(x_{j}\right) \leq r$, where the sum means the supremum of all finite partial sums. To see this, let $\Phi$ be the set of finite subsets of $J$. If $x \in C$, then $\sum_{j \in F} x_{j} \in Q$ for each $F \in \Phi\left(\right.$ as $\left.\sum_{j \in F} q_{j}\left(x_{j}\right) \leq \sum_{j \in J} q_{j}\left(x_{j}\right) \leq r\right)$ and thus $x \in \bar{Q}$.

Conversely, let $x=\left(x_{j}\right)_{j \in J} \in \prod_{j \in J} E_{j}$ with $x \notin C$; thus $\sum_{j \in J} q_{j}\left(x_{j}\right)>r$. There exists a finite subset $F \subseteq J$ such that $\sum_{j \in F} q_{j}\left(x_{j}\right)>r$. Since

$$
\left\{\left(y_{j}\right)_{j \in J} \in \prod_{j \in J} E_{j}: \sum_{j \in F} q_{j}\left(y_{j}\right)>r\right\}
$$

is an open subset of $\prod_{j \in J} E_{j}$ that has empty intersection with $Q$, we have $x \notin \bar{Q}$. Thus $\bar{Q}=C$.

By the preceding, $\left(\oplus_{j \in J} E_{j}\right) \cap \bar{Q}=\left(\oplus_{j \in J} E_{j}\right) \cap C=Q$. Since $L$ is closed in $\prod_{j \in J} E_{j}$ and contains $Q$, we have $\bar{Q} \subseteq L$. Now $K=\prod_{j \in J} K_{j}$ is a closed subset of $P=\prod_{j \in J} G_{j}$ and

$$
\psi:=\prod_{j \in J}\left(\left.\phi_{j}\right|_{K_{j}}\right): K \longrightarrow L, \quad\left(x_{j}\right)_{j \in J} \longmapsto\left(\phi_{j}\left(x_{j}\right)\right)_{j \in J}
$$

is a homeomorphism, whence $\psi^{-1}(\bar{Q})$ is closed in $K$ and hence also closed in $P=$ $\prod_{j \in J} G_{j}$. 
Let $\bar{W}$ be the closure of $W$ in $P$. By the preceding, $\bar{W} \subseteq \psi^{-1}(\bar{Q})$ and thus $\bar{W}=$ $\psi^{-1}(\bar{Q})$, as $\psi$ is a homeomorphism. For $x \in K$, we have $\psi(x) \in \oplus_{j \in J} E_{j}$ if and only if $x \in \oplus_{j \in J} G_{j}$. Hence,

$$
G \cap \bar{W}=G \cap \psi^{-1}(\bar{Q})=\psi^{-1}\left(\left(\bigoplus_{j \in J} E_{j}\right) \cap \bar{Q}\right)=\psi^{-1}(Q)=W \subseteq S,
$$

entailing that Lemma 5.1 (iii) is also satisfied.

\section{Completeness of Diffeomorphism Groups}

The next proposition is used to prove completeness of diffeomorphism groups.

Proposition 6.1 Let $G$ be a topological group that is the projective limit of a projective sequence $\left(\left(G_{n}\right)_{n \in \mathbb{N}},\left(q_{n, m}\right)_{n \leq m}\right)$ of topological Hausdorff groups. Assume that $G_{n}$ admits a $C^{1}$-manifold structure modelled on a Banach space $\left(E_{n},\|\cdot\|_{n}\right)$ for each $n \in \mathbb{N}$, and assume that for each $n \in \mathbb{N}$, there exists $m \geq n$ such that the map

$$
\mu_{n, m}: G_{m} \times G_{m} \longrightarrow G_{n}, \quad(x, y) \longmapsto q_{n, m}(x y)=q_{n, m}(x) q_{n, m}(y)
$$

is $C^{1}$. Then $G$ is a complete topological group.

Proof For $n \in \mathbb{N}$, let $q_{n}: G \rightarrow G_{n}$ be the limit map. Let $\left(x_{\alpha}\right)_{\alpha \in A}$ be a left Cauchy net in G. By Lemma 2.4, it suffices to show that, for each $n \in \mathbb{N}$, the left Cauchy net $\left(q_{n}\left(x_{\alpha}\right)\right)_{\alpha \in A}$ converges in $G_{n}$. By hypothesis, $\mu_{n, m}$ is $C^{1}$ for some $m \geq n$. We write $\|\cdot\|$ for the norm $E_{n} \times E_{n} \rightarrow\left[0, \infty\left[,(x, y) \mapsto \max \left\{\|x\|_{n},\|y\|_{n}\right\}\right.\right.$. Let $\phi: U \rightarrow V$ be a $C^{1}$-diffeomorphism from an open identity neighbourhood $U \subseteq G_{m}$ onto an open 0 -neighbourhood $V \subseteq E_{m}$ and $\psi: P \rightarrow Q$ be a $C^{1}$-diffeomorphism from an open identity neighbourhood $P \subseteq G_{n}$ onto an open 0 -neighbourhood $Q \subseteq E_{n}$, such that $\phi(e)=0$ and $\psi(e)=0$. After shrinking $U$, we may assume that $\mu_{n, m}(U \times U) \subseteq P$, enabling us to consider the $C^{1}$-map

$$
f:=\psi \circ \mu_{n, m} \circ\left(\phi^{-1} \times \phi^{-1}\right): V \times V \longrightarrow Q, \quad(x, y) \longmapsto \psi\left(q_{n, m}\left(\phi^{-1}(x) \phi^{-1}(y)\right)\right) .
$$

After shrinking $V$, we may assume that $f$ is Lipschitz (Lemma 2.2). Let $L \geq 0$ be a Lipschitz constant for $f$. Then $\|f(x, y)-f(x, 0)\|_{n} \leq L\|(0, y)\|=L\|y\|_{n}$ for all $(x, y) \in V \times V$. Let $C$ be a closed 0 -neighbourhood in $E_{n}$ such that $C \subseteq Q$. Then $q_{n}^{-1}\left(\psi^{-1}(C)\right) \cap q_{m}^{-1}(U)$ is an identity neighbourhood in $G$. There is $\alpha_{0} \in A$ such that $x_{\beta}^{-1} x_{\alpha} \in q_{n}^{-1}\left(\psi^{-1}(C)\right) \cap q_{m}^{-1}(U)$ for all $\alpha, \beta \geq \alpha_{0}$. Thus $z_{\alpha}:=x_{\alpha_{0}}^{-1} x_{\alpha} \in q_{n}^{-1}\left(\psi^{-1}(C)\right) \cap$ $q_{m}^{-1}(U)$ for all $\alpha \geq \alpha_{0}$, and $\left(q_{n}\left(z_{\alpha}\right)\right)_{\alpha \geq \alpha_{0}}$ is a left Cauchy net in $G_{n}$, noting that

$$
z_{\beta}^{-1} z_{\alpha}=x_{\beta}^{-1} x_{\alpha} \in q_{m}^{-1}(U) \quad \text { for all } \alpha, \beta \geq \alpha_{0} .
$$

If we can show that $\left(q_{n}\left(z_{\alpha}\right)\right)_{\alpha \geq \alpha_{0}}$ converges in $G_{n}$, then also the subnet $\left(q_{n}\left(x_{\alpha}\right)\right)_{\alpha \geq \alpha_{0}}$ of $\left(q_{n}\left(x_{\alpha}\right)_{\alpha \in A}\right)$ will converge, as $q_{n}\left(x_{\alpha}\right)=q_{n}\left(x_{\alpha_{0}}\right) q_{n}\left(z_{\alpha}\right)$. Hence, $\left(q_{n}\left(x_{\alpha}\right)\right)_{\alpha \in A}$ will converge. Since $z_{\alpha}=z_{\beta}\left(z_{\beta}^{-1} z_{\alpha}\right)$ and $z_{\beta}=z_{\beta}$ e, we see that

$$
\psi\left(q_{n}\left(z_{\alpha}\right)\right)-\psi\left(q_{n}\left(z_{\beta}\right)\right)=f\left(q_{m}\left(z_{\beta}\right), q_{m}\left(z_{\beta}^{-1} z_{\alpha}\right)\right)-f\left(q_{m}\left(z_{\beta}\right), 0\right)
$$

and thus $\left\|\psi\left(q_{n}\left(z_{\alpha}\right)\right)-\psi\left(q_{n}\left(z_{\beta}\right)\right)\right\|_{n} \leq L\left\|q_{m}\left(z_{\beta}^{-1} z_{\alpha}\right)\right\|_{n}$, which can be made arbitrarily small for large $\alpha, \beta$. Hence, $\left(\psi\left(q_{n}\left(z_{\alpha}\right)\right)\right)_{\alpha \geq \alpha_{0}}$ is a Cauchy net in $\left(E_{n},+\right)$ and thus convergent to some $w \in E_{n}$. Since $w_{\alpha}:=\psi\left(q_{n}\left(z_{\alpha}\right)\right) \in C$ for all $\alpha \geq \alpha_{0}$ and $C$ is closed 
in $E$, we deduce that $w \in C \subseteq Q$. As a consequence, $q_{n}\left(z_{\alpha}\right)=\psi^{-1}\left(w_{\alpha}\right)$ converges to $\psi^{-1}(w)$.

Remark 6.2 (a) In particular, Proposition 6.1 shows that every strong (ILB)-Lie group (as in [38]) is complete.

(b) Since $\operatorname{Diff}(M)$ is a strong (ILB)-Lie group for each compact smooth manifold $M$ without boundary (see [38]), we deduce from (a) that $\operatorname{Diff}(M)$ is complete.

(c) Let $M$ be a $\sigma$-compact finite-dimensional smooth manifold (without boundary). If $K \subseteq M$ is a compact subset, then

$$
\operatorname{Diff}_{K}(M):=\left\{\phi \in \operatorname{Diff}_{c}(M):(\forall x \in M \backslash K) \phi(x)=x\right\}
$$

is a Lie subgroup of $\operatorname{Diff}_{c}(M)$. Morse Theory [27] provides a compact submanifold $N \subseteq M$ with smooth boundary such that $K$ is contained in the interior of $N$. Let $N^{*}$ be the double of $N$, which is a compact smooth manifold without boundary obtained by glueing two copies of $N$ along their boundary. ${ }^{11}$ Then $\operatorname{Diff}_{K}(M) \cong \operatorname{Diff}_{K}\left(N^{*}\right)$ as a Lie group. As Diff ${ }_{K}\left(N^{*}\right)$ is a closed subgroup of the complete topological group $\operatorname{Diff}\left(N^{*}\right)$, we see that $\operatorname{Diff}_{K}\left(N^{*}\right)$, and hence also $\operatorname{Diff}_{K}(M)$, is complete.

(d) Using Theorem 1.1, we now obtain completeness of $\operatorname{Diff}_{c}(M)$ for $\sigma$-compact $M$, as described in the Introduction; applying Theorem 1.2 to an open subgroup, completeness of $\operatorname{Diff}_{c}(M)$ for paracompact $M$ follows.

\section{Completeness of Mapping Groups}

We now discuss completeness of mapping groups and test function groups.

\subsection{Spaces of $C^{k}$-maps Between Manifolds}

If $k \in \mathbb{N} \cup\{\infty\}$ and $M$ is a $C^{k}$-manifold (possibly with boundary) modelled on a Hausdorff locally convex space, we let $T M$ be the tangent bundle and recursively define $T^{j+1} M:=T\left(T^{j} M\right)$ for $j \in \mathbb{N}$ such that $j \leq k$. If $f: M \rightarrow N$ is a $C^{k}$-map to another such manifold, we let $T f: T M \rightarrow T N$ be the tangent map and recursively set $T^{j} f:=T\left(T^{j-1} f\right): T^{j} M \rightarrow T^{j} N$ for all $2 \leq j \in \mathbb{N}$ such that $j \leq k$. For convenience, $T^{0} M:=M$ and $T^{0} f:=f$. We endow the set $C^{k}(M, N)$ of all $C^{k}$-maps from $M$ to $N$ with the so-called compact-open $C^{k}$-topology, i.e., the initial topology with respect to the mappings $T^{j}: C^{k}(M, N) \rightarrow C\left(T^{j} M, T^{j} N\right), f \mapsto T^{j} f$ for $j \in \mathbb{N}_{0}$ such that $j \leq k$, where $C\left(T^{j} M, T^{j} N\right)$ is endowed with the compact-open topology [37].

\subsection{Topological Groups of $C^{k}$-maps}

If $H$ is a Lie group, with multiplication $\mu: H \times H \rightarrow H$, then the tangent map

$$
T \mu: T(H \times H) \longrightarrow T H
$$

makes $T H$ a Lie group, if we identify $T(H \times H)$ with $T H \times T H$ in the usual way. Then $C^{k}(M, H)$ is a group for $M$ as before and $k \in \mathbb{N}_{0} \cup\{\infty\}$, with pointwise product

\footnotetext{
${ }^{11}$ Our use of the double was stimulated by discussions in [42].
} 
$f g:=\mu \circ(f, g)$ for $f, g \in C^{k}(M, H)$. If $k \geq 1$, then

$$
T(f g)=T \mu \circ(T f, T g)=T f T g
$$

is the product in $C(T M, T H)$, whence $T f$ is a group homomorphism and hence also $T^{j} f$ for all $j \in \mathbb{N}_{0}$ such that $j \leq k$. Since $C\left(T^{j} M, T^{j} H\right)$ is a topological group for each $j$, we deduce that $C^{k}(M, H)$ is a topological group [37].

Our first goal is to establish completeness properties for the topological groups $C^{k}(M, H)$.

Proposition 7.1 Let H be a Lie group modelled on a locally convex Hausdorff space E and let $M$ be a finite-dimensional $C^{k}$-manifold (possibly with boundary) for some $k \epsilon$ $\mathbb{N}_{0} \cup\{\infty\}$. If $H$ and $E$ are complete, then the topological group $C^{k}(M, H)$ is also complete.

The proof is based on two lemmas.

Lemma 7.2 Given $k \in \mathbb{N}$, let $M$ be a finite-dimensional $C^{k}$-manifold (possibly with boundary) and $N$ be a $C^{k}$-manifold. Then the map

$$
\theta: C^{k}(M, N) \longrightarrow C(M, N) \times C^{k-1}(T M, T N), \quad f \longmapsto(f, T f)
$$

is a topological embedding with closed image. If $N$ is a Lie group, then $\theta$ is a homomorphism of groups.

Proof The final observation follows from (7.1). It is clear that $\theta$ is injective. Moreover, the topology $\mathcal{O}$ on $C^{k}(M, N)$ making $\theta$ a topological embedding is initial with respect to the inclusion maps

$$
T^{0}: C^{k}(M, N) \rightarrow C(M, N) \quad \text { and } \quad T: C^{k}(M, N) \rightarrow C^{k-1}(T M, T N) .
$$

As the topology on $C^{k-1}(T M, T N)$ is initial with respect to the maps

$$
T^{j}: C^{k-1}(T M, T N) \rightarrow C\left(T^{j+1} M, T^{j+1} N\right) \text { for } j \in\{0, \ldots, k-1\},
$$

we deduce, using the well-known transitivity of initial topologies, that $\mathcal{O}$ is initial with respect to $T^{0}$ and the maps

$$
T^{j} \circ T: C^{k}(M, N) \rightarrow C\left(T^{j+1} M, T^{j+1} N\right) \text { for } j \in\{0, \ldots, k-1\} .
$$

Hence, $\mathcal{O}$ coincides with the compact-open $C^{k}$-topology.

To see that $\theta$ has a closed image, let $\left(f_{\alpha}, T f_{\alpha}\right)_{\alpha \in A}$ be a net in the image of $\theta$ that converges to some $(f, g) \in C(M, N) \times C^{k-1}(T M, T N)$. It now suffices to show that each $x \in M$ has an open neighbourhood $U$ such that $\left.f\right|_{U}$ is $C^{1}$ and $T\left(\left.f\right|_{U}\right)=\left.g\right|_{T U}$; then $f$ is $C^{k}$ and $g=T f$, whence $(f, g)=\theta(f)$ is in the image of $\theta$.

For $x \in M$, there is a chart $\psi: U_{\psi} \rightarrow V_{\psi} \subseteq Y$ of $N$ such that $f(x) \in U_{\psi}$, where $Y$ is the modelling space of $N$, and a chart $\phi: U_{\phi} \rightarrow V_{\phi} \subseteq X$ of $M$ with $x \in U_{\phi}$ such that $U_{\phi}$ has compact closure $K:=\overline{U_{\phi}}$ and $f\left(\overline{U_{\phi}}\right) \subseteq U_{\psi}$, where $X$ is the modelling space of $M$. As the compact-open $C^{k}$-topology on $C^{k}(M, N)$ is finer than the compactopen topology, the set $W:=\left\{h \in C^{k}(M, N): h(K) \subseteq U_{\psi}\right\}$ is an open neighbourhood of $f$ in $C^{k}(M, N)$. Thus, we find $\alpha_{0} \in A$ such that $f_{\alpha} \in W$ for all $\alpha \in A$ such that $\alpha \geq \alpha_{0}$. For such $\alpha$, we can define $h_{\alpha}:=\psi \circ f_{\alpha} \circ \phi^{-1}: V_{\phi} \rightarrow Y$. Then $h_{\alpha} \rightarrow \psi \circ f \circ \phi^{-1}$ and 
$\left.d\left(h_{\alpha}\right) \rightarrow d \psi \circ g\right|_{T U_{\phi}} \circ T \phi^{-1}$ uniformly on compact sets, entailing that $h:=\psi \circ f \circ \phi^{-1}$ is $C^{1}$ with $d h=\left.d \psi \circ g\right|_{T U_{\phi}} \circ T \phi^{-1}$, and thus $T h=\left.T \psi \circ g\right|_{T U_{\phi}} \circ T \phi^{-1}$. Hence $\left.f\right|_{U_{\phi}}$ is $C^{1}$ with $T\left(\left.f\right|_{U_{\phi}}\right)=\left.g\right|_{T U_{\phi}}$.

Lemma 7.3 Let $M$ and $N$ be smooth manifolds (possibly with boundary), both modelled on Hausdorff locally convex spaces. Then

$$
C^{\infty}(M, N)=\lim _{k \in \mathbb{N}_{0}} C^{k}(M, N)
$$

as a topological space, using the respective inclusion maps as the bonding maps and limit maps.

Proof Consider the standard realization $P \subseteq \prod_{k \in \mathbb{N}_{0}} C^{k}(M, N)$ of the projective limit. As all bonding maps are the inclusion maps, it is the set of all sequences

$$
\left(f_{k}\right)_{k \in \mathbb{N}_{0}} \in \prod_{k \in \mathbb{N}_{0}} C^{k}(M, N)
$$

such that $f_{j}=f_{k}$, for all $j, k \in \mathbb{N}_{0}$, such that $j \leq k$. Then $f_{0}=f_{k}$, for all $k \in \mathbb{N}_{0}$, and thus $f_{0} \in C^{\infty}(M, N)$, entailing that the map $\Phi: C^{\infty}(M, N) \rightarrow P, f \mapsto(f)_{k \in \mathbb{N}_{0}}$ is a bijection. The topology $\mathcal{O}$ on $C^{\infty}(M, N)$, making $\Phi$ a homeomorphism, is initial with respect to the compositions $T^{j} \circ \pi_{k} \circ \Phi=T^{j}$ for $k \in \mathbb{N}_{0}$ and $j \in \mathbb{N}_{0}$ such that $j \leq k$, where $\pi_{k}$ is the projection from the direct product onto its $k$-th factor. It therefore coincides with the compact-open $C^{\infty}$-topology.

Proof of Proposition 7.1 If we can show that $C^{k}(M, H)$ is complete for each $k \in \mathbb{N}_{0}$, then also $C^{\infty}(M, H)$ (which is the projective limit of the latter topological groups by Lemma 7.3) will be complete. We proceed by induction. If $k=0$, then $C^{0}(M, H)=$ $C(M, H)$ is complete since $H$ is complete and $M$, being locally compact, is a $k_{\mathbb{R}^{-}}$ space $^{12}$.

If $k \in \mathbb{N}$ and the assertion holds for $k-1$ in place of $k$, then $C^{k-1}(T M, T H)$ is complete as $T M$ has finite dimension, $T H \cong L(H) \rtimes H$ is complete (see Proposition $2.3(\mathrm{v}))$ and also its modelling space $E \times E$ is complete. Moreover, $C(M, H)$ is complete. As, by Lemma 7.2, the topological group $C^{k}(M, H)$ is isomorphic to a closed subgroup of the direct product $C(M, H) \times C^{k-1}(M, T H)$ of complete groups, also $C^{k}(M, H)$ is complete.

Remark 7.4 (i) If $M$ is a compact smooth manifold and $H$ a Lie group, then the topology on the Lie group $C^{k}(M, H)$ (for $k \in \mathbb{N}_{0} \cup\{\infty\}$ ) coincides with the compactopen $C^{k}$-topology defined above.Hence $C^{k}(M, H)$ is complete whenever $H$ and its modelling space are complete.

(ii) If $M$ is a finite-dimensional $\sigma$-compact smooth manifold and $K \subseteq M$ a compact subset, then the topology on the Lie group

$$
C_{K}^{k}(M, H):=\left\{\gamma \in C^{k}(M, H): \operatorname{supp}(\gamma) \subseteq K\right\}
$$

\footnotetext{
${ }^{12}$ Recall that a topological space $X$ is called a $k_{\mathbb{R}}$-space if it is Hausdorff and functions $f: X \rightarrow \mathbb{R}$ are continuous if and only if $\left.f\right|_{K}$ is continuous for each compact subset $K \subseteq X$.
} 
is induced by the compact-open $C^{k}$-topology on $C^{k}(M, H)$. Since $C_{K}^{k}(M, H)$ is a closed subgroup of $C^{k}(M, H)$, we deduce that $C_{K}^{k}(M, H)$ is complete whenever $H$ and its modelling space are complete.

Proposition 7.5 Let $M$ be a paracompact finite-dimensional smooth manifold and let $H$ be a Lie group. If $H$ and its modelling space are complete, then $C_{c}^{k}(M, H)$ is complete for each $k \in \mathbb{N}_{0} \cup\{\infty\}$.

Proof If $M$ is $\sigma$-compact, we choose a sequence $\left(K_{n}\right)_{n \in \mathbb{N}}$ of compact subsets of $M$ such that $M=\bigcup_{n \in \mathbb{N}} K_{n}$ and $K_{n} \subseteq K_{n+1}^{0}$ for each $n$. Then

$$
C_{K_{1}}^{k}(M, H) \subseteq C_{K_{2}}^{k}(M, H) \subseteq \cdots
$$

is a strict direct sequence of topological groups and product sets are large in

$$
C_{c}^{k}(M, H)=\bigcup_{n \in \mathbb{N}} C_{K_{n}}^{k}(M, H)
$$

as the product map

$$
\bigoplus_{n \in \mathbb{N}} C_{K_{n}}^{k}(M, H) \longrightarrow C_{c}^{k}(M, H), \quad\left(\gamma_{1}, \ldots, \gamma_{n}, e, e, \cdots\right) \longmapsto \gamma_{1} \gamma_{2} \cdots \gamma_{n}
$$

admits a smooth local section around $e$ which takes $e$ to $e$ [21, Example 11.6, Remark 11.5]. Since $C_{K_{n}}^{k}(M, H)$ is complete for each $n \in \mathbb{N}$ (Remark 7.4 (ii)), we deduce with Theorem 1.1 that $C_{c}^{k}(M, H)$ is complete.

If $M$ is merely paracompact, we let $\left(M_{j}\right)_{j \in J}$ be the family of connected components of $M$ (each of which is $\sigma$-compact). Then the map

$$
\Phi: C_{c}^{k}(M, H) \longrightarrow \bigoplus_{j \in J} C_{c}^{k}\left(M_{j}, H\right), \quad \gamma \longmapsto\left(\left.\gamma\right|_{M_{j}}\right)_{j \in J}
$$

is an isomorphism of groups and we give $C_{c}^{k}(M, H)$ the smooth Lie group structure that turns $\Phi$ into an isomorphism of Lie groups. As the weak direct product is complete by the first part of the proof and Theorem 1.2, we see that $C_{c}^{k}(M, H)$ is also complete.

Remark 7.6 Let $H$ be a Lie group, $M$ be a smooth manifold of dimension $m \in \mathbb{N}$, and $P \rightarrow M$ be a smooth principal bundle with structure group $H$.

(i) If $M$ is $\sigma$-compact and the condition $\mathrm{SUB}_{\oplus}$ of [41] is satisfied, ${ }^{13}$ then the gauge group $\operatorname{Gau}_{c}(P)$ of $P$ is a Lie group that is isomorphic to a closed Lie subgroup of the weak direct product $\oplus_{n \in \mathbb{N}} C^{\infty}\left(K_{n}, H\right)$, where $\left(K_{n}\right)_{n \in \mathbb{N}}$ is a locally finite cover of $M$ by $m$-dimensional compact smooth submanifolds $K_{n}$ with boundary such that $P$ is trivializable on some open neighbourhood of $K_{n}$. If $H$ and its modelling space are complete, then $C^{\infty}\left(K_{n}, H\right)$ is complete for each $n \in \mathbb{N}$ (by Proposition 7.1), whence also the weak direct product is complete (by Theorem 1.2) and hence also $\operatorname{Gau}_{c}(P)$, being isomorphic to a closed subgroup of the latter as a topological group. Then the full group $\operatorname{Aut}_{c}(P)$ of compactly supported symmetries of $P$ is complete ${ }^{14}$, as it is an

\footnotetext{
${ }^{13}$ This is automatic if $H$ is locally exponential in the sense that $H$ has a smooth exponential function that is a local $C^{\infty}$-diffeomorphism at 0 .

${ }^{14}$ In [41], $\operatorname{Aut}_{c}(P)$ was made into a Lie group. For compact $M$, the Lie group $\operatorname{Aut}(P)$ was already constructed in [45].
} 
extension

$$
\{e\} \rightarrow \operatorname{Gau}_{c}(P) \rightarrow \operatorname{Aut}_{c}(P) \rightarrow \operatorname{Diff}_{c}(M)_{P} \rightarrow\{e\}
$$

of Lie groups (and hence of topological groups) for some open subgroup $\operatorname{Diff}(M)_{P} \subseteq$ $\operatorname{Diff}_{c}(M)$. Since $\operatorname{Diff}_{c}(M)$ is complete (as already observed) and also $\operatorname{Gau}_{c}(P)$ is complete, so is the extension $\operatorname{Aut}_{c}(P)$ (as recalled in Proposition 2.3(iv)).

(ii) If $M$ is paracompact and condition $\mathrm{SUB}_{\oplus}$ is satisfied by $\left.P\right|_{C}$ for each connected component $C$ of $M$, let $\left(M_{j}\right)_{j \in J}$ be the family of connected components of $M$. We can identify $\operatorname{Gau}_{c}(P)$ with the weak direct product $\oplus_{j \in J} \operatorname{Gau}_{c}\left(M_{j}\right)$ (whence it can be considered as a complete Lie group by (i) and Theorem 1.2). Moreover, $\operatorname{Aut}_{c}(P)$ can be made a Lie group having the weak direct product $\bigoplus_{j \in J} \operatorname{Aut}_{c}\left(\left.P\right|_{M_{j}}\right)$ as an open subgroup. Hence $\operatorname{Aut}_{c}(P)$ is complete, using Theorem 1.2.

\section{Product Sets in Unions of Banach-Lie Groups}

We now discuss ascending unions of Banach-Lie groups.

\subsection{Well-behaved Ascending Unions}

Let $G_{1} \subseteq G_{2} \subseteq \cdots$ be analytic Banach-Lie groups over $\mathbb{K} \in\{\mathbb{R}, \mathbb{C}\}$ such that the inclusion maps $j_{n+1, n}: G_{n} \rightarrow G_{n+1}$ are $\mathbb{K}$-analytic group homomorphisms. Identifying the Banach-Lie algebra $\mathfrak{g}_{n}:=L\left(G_{n}\right)$ with the image of the map $L\left(j_{n+1, n}\right)$ in $\mathfrak{g}_{n+1}$, we can consider the ascending union $\mathfrak{g}:=\bigcup_{n \in \mathbb{N}} \mathfrak{g}_{n}$ and endow it with the locally convex direct limit topology. Give $G:=\cup_{n \in \mathbb{N}} G_{n}$ the unique group structure making each inclusion map $G_{n} \rightarrow G$ a group homomorphism. Define $\exp _{G}: \mathfrak{g} \rightarrow G$ piecewise via $\exp _{G}(x):=\exp _{G_{n}}(x)$ if $x \in \mathfrak{g}_{n}$.

Lemma 8.1 (Dahmen's setting) If, in the preceding situation,

(i) $\mathfrak{g}$ is Hausdorff,

(ii) there are norms $\|\cdot\|_{n}$ on $\mathfrak{g}_{n}$ defining its topology for $n \in \mathbb{N}$, such that the Lie bracket of $\mathfrak{g}_{n}$ and each inclusion map $\left(\mathfrak{g}_{n},\|\cdot\|_{n}\right) \rightarrow\left(\mathfrak{g}_{n+1},\|\cdot\|_{n+1}\right)$ has operator norm $\leq 1$,

(iii) $\exp _{G}$ is injective on some 0-neighbourhood,

then $G$ admits a unique $\mathbb{K}$-analytic Lie group structure such that $P:=\exp _{G}(Q)$ is open in $G$ for some open 0 -neighbourhood $Q \subseteq \mathfrak{g}$ and $\left.\exp _{G}\right|_{Q} ^{P}$ a diffeomorphism of $\mathbb{K}$-analytic manifolds. (See [12, Theorem C]).

Proposition 8.2 Let $G_{1} \subseteq G_{2} \subseteq \cdots$ be analytic Banach-Lie groups over $\mathbb{K} \in\{\mathbb{R}, \mathbb{C}\}$ such that the inclusion maps $G_{n} \rightarrow G_{n+1}$ are $\mathbb{K}$-analytic group homomorphisms. Assume that Dahmen's conditions (i)-(iii) from 8.1 are satisfied and endow $G$ with the $\mathbb{K}$-analytic Lie group structure described there. Let $\mathcal{O}$ be the topology on the Lie group $G$. Then product sets are large in $(G, \mathcal{O})=\cup_{n \in \mathbb{N}} G_{n}$. As a consequence, $\mathcal{O}=\mathcal{O}_{T G}$ holds, i.e., $\mathcal{O}$ makes $G$ the direct limit topological group $\lim _{\longrightarrow} G_{n}$. If, moreover, the direct sequence $G_{1} \subseteq G_{2} \subseteq \cdots$ is strict, then $(G, \mathcal{O})$ is complete.

Before we prove Proposition 8.2, let us compile useful facts concerning the BakerCampbell-Hausdorff (BCH) multiplication. 
Completeness of Infinite-dimensional Lie Groups in Their Left Uniformity

\subsection{BCH Multiplication}

Let $\mathfrak{g}$ be a Banach-Lie algebra and $\|\cdot\|$ a norm on $\mathfrak{g}$ that is compatible in the sense that it defines the topology of $\mathfrak{g}$ and $\|[x, y]\| \leq\|x\|\|y\|$ holds for all $x, y \in \mathfrak{g}$. Then the $\mathrm{BCH}$ series converges for $x, y \in \mathfrak{g}$ with $\|x\|+\|y\|<\ln \frac{3}{2}$ and defines an analytic function

$$
\{(x, y) \in \mathfrak{g} \times \mathfrak{g}:\|x\|+\|y\|<\ln (3 / 2)\} \longrightarrow B_{\ln 2}^{\mathfrak{g}}(0), \quad(x, y) \longmapsto x * y
$$

(see [10]). If $\mathfrak{g}=L(G)$ for some Banach-Lie group $G$, then

$$
\exp _{G}(x * y)=\exp _{G}(x) \exp _{G}(y), \quad \text { for all } x, y \in \mathfrak{g} \text { with }\|x\|+\|y\|<\ln \frac{3}{2} .
$$

See [13, Lemma 3.5 (a)] for the following estimates concerning derivatives of the $\mathrm{BCH}$ multiplication.

Lemma 8.3 There exists $\left.s_{0} \in\right] 0, \frac{1}{3} \ln \frac{3}{2}[$ such that, for each Banach-Lie algebra $\mathfrak{g}$ and compatible norm $\|\cdot\|$ on $\mathfrak{g}$,

$$
\left(\forall x, y \in B_{s_{0}}^{\mathfrak{g}}(0)\right)\left\|\left(\mu^{\mathfrak{g}}\right)^{\prime}(x, y)-\alpha^{\mathfrak{g}}\right\|_{\mathrm{op}} \leq \frac{1}{2},
$$

where $\alpha^{\mathfrak{g}}: \mathfrak{g} \times \mathfrak{g} \rightarrow \mathfrak{g},(x, y) \mapsto x+y$ is addition and

$$
\mu^{\mathfrak{g}}: B_{s_{0}}^{\mathfrak{g}}(0) \times B_{s_{0}}^{\mathfrak{g}}(0) \longrightarrow \mathfrak{g}, \quad(x, y) \longmapsto x * y
$$

is the BCH multiplication.

To calculate the operator norm, the maximum norm was used on $\mathfrak{g} \times \mathfrak{g}$ here. With $s_{0}$ and notation as in Lemma 8.3, we deduce the following.

Lemma 8.4 For each Banach-Lie algebra $\mathfrak{g}$ and compatible norm $\|\cdot\|$ on $\mathfrak{g}$, we have

$$
x * y+B_{r / 2}^{\mathfrak{g}}(0) \subseteq x * B_{r}^{\mathfrak{g}}(y) \subseteq x * y+B_{3 r / 2}^{\mathfrak{g}}(0)
$$

for all $x \in B_{s_{0}}^{\mathfrak{g}}(0), y \in B_{s_{0} / 2}^{\mathfrak{g}}(0)$, and $\left.\left.r \in\right] 0, \frac{s_{0}}{2}\right]$.

Proof Setting $R(x, y):=\mu^{\mathfrak{g}}(x, y)-x-y$, we have $\mu^{\mathfrak{g}}(x, y)=x+y+R(x, y)$ for all $x, y \in B_{s_{0}}^{\mathfrak{g}}(0)$. Since $\left\|R^{\prime}(x, y)\right\|_{\text {op }} \leq \frac{1}{2}$, for all $(x, y) \in B_{s_{0}}^{\mathfrak{g}}(0) \times B_{s_{0}}^{\mathfrak{g}}(0)$ by $(8.2)$, and the latter set is convex, Lemma 2.2 (ii) shows that $\operatorname{Lip}(R) \leq \frac{1}{2}$. For $x \in B_{s_{0}}^{\mathfrak{g}}(0)$, consider the map $\mu_{x}^{\mathfrak{g}}: B_{s_{0}}^{\mathfrak{g}}(0) \rightarrow \mathfrak{g}, y \mapsto \mu^{\mathfrak{g}}(x, y)$. For all $y, z \in B_{s_{0}}^{\mathfrak{g}}(0)$, we have

$$
\begin{aligned}
\left\|\mu_{x}^{\mathfrak{g}}(z)-\mu_{x}^{\mathfrak{g}}(y)-\mathrm{id}_{\mathfrak{g}}(z-y)\right\| & =\left\|\mu^{\mathfrak{g}}(x, z)-(x+z)-\mu^{\mathfrak{g}}(x, y)+x+y\right\| \\
& =\|R(x, z)-R(x, y)\| \leq \operatorname{Lip}(R)\|z-y\|,
\end{aligned}
$$

and thus $\operatorname{Lip}\left(\mu_{x}^{\mathfrak{g}}-\operatorname{id}_{\mathfrak{g}}\right) \leq \operatorname{Lip}(R) \leq \frac{1}{2}$. Applying now the Quantitative Inverse Function Theorem [20, Lemma 6.1 (a)] (or the version in [44]), to the function $\mu_{x}^{\mathfrak{g}}$ with $A:=\mathrm{id}_{\mathfrak{g}}$, we get (8.3).

Proof of Proposition 8.2 To see that product sets are large in $G=\cup_{n \in \mathbb{N}} G_{n}$, let $\left(U_{n}\right)_{n \in \mathbb{N}}$ be a sequence of identity neighbourhoods $U_{n} \subseteq G_{n}$. By hypothesis,

$$
\|x\|_{m} \leq\|x\|_{k} \quad \text { for all integers } 1 \leq k \leq m \text { and all } x \in \mathfrak{g}_{k} .
$$


Let $s_{0}$ be as in Lemma 8.3. For $n \in \mathbb{N}$, choose $\left.r_{n} \in\right] 0, s_{0} / 2^{n+1}[$ so small that

$$
V_{n}:=\exp _{G_{n}}\left(B_{r_{n}}^{\mathfrak{g}_{n}}(0)\right) \subseteq U_{n} .
$$

For $x, y \in B_{s_{0}}^{\mathfrak{g}_{n}}(0)$, write $x *_{n} y:=\mu^{\mathfrak{g}_{n}}(x, y)$ for the BCH multiplication, (as in Lemma 8.3). Define $W_{1}:=B_{r_{1}}^{\mathfrak{g}_{1}}(0)$. We claim that $W_{n}:=W_{n-1} *_{n} B_{r_{n}}^{\mathfrak{g}_{n}}(0)$ can be defined for each integer $n \geq 2$, and

$$
\sum_{k=1}^{n} B_{r_{k} / 2}^{\mathfrak{g}_{k}}(0) \subseteq W_{n} \subseteq \sum_{k=1}^{n} B_{3 r_{k} / 2}^{\mathfrak{g}_{k}}(0) .
$$

If the claim is true, then $W:=\bigcup_{n \in \mathbb{N}} W_{n}$ is a 0-neighbourhood in $\mathfrak{g}$, as it contains the convex set $S:=\bigcup_{n \in \mathbb{N}}\left(B_{r_{1} / 2}^{\mathfrak{g}_{1}}(0)+\cdots+B_{r_{n} / 2}^{\mathfrak{g}_{n}}(0)\right)$ that is an open 0-neighbourhood in the locally convex direct limit $\mathfrak{g}=\bigcup_{n \in \mathbb{N}} \mathfrak{g}_{n}$ as it intersects each $\mathfrak{g}_{n}$ in an open 0 -neighbourhood. Since $\exp _{G}(W)$ contains the open subset $\exp _{G}(S \cap Q)$ of $G$ (with $Q$ as in Lemma 8.1), we deduce that $\exp _{G}(W)$ is an identity neighbourhood in $G$. Now $\exp _{G}\left(W_{n}\right)=V_{1} V_{2} \cdots V_{n}$, for each $n \in \mathbb{N}$; this is trivial if $n=1$ and follows inductively as

$$
\begin{aligned}
\exp _{G}\left(W_{n}\right) & =\exp _{G_{n}}\left(W_{n}\right)=\exp _{G_{n}}\left(W_{n-1} *_{n} B_{r_{n}}^{\mathfrak{g}_{n}}(0)\right) \\
& =\exp _{G_{n}}\left(W_{n-1}\right) \exp _{G_{n}}\left(B_{r_{n}}^{\mathfrak{g}_{n}}(0)\right)=\exp _{G_{n-1}}\left(W_{n-1}\right) V_{n} \\
& =V_{1} \cdots V_{n-1} V_{n},
\end{aligned}
$$

using (8.1), the definition of $V_{n}$, and the inductive hypothesis. Thus

$$
U:=\bigcup_{n \in \mathbb{N}} U_{1} \cdots U_{n} \supseteq \bigcup_{n \in \mathbb{N}} V_{1} \cdots V_{n}=\bigcup_{n \in \mathbb{N}} \exp _{G}\left(W_{n}\right)=\exp _{G}(W),
$$

whence $U$ is an identity neighbourhood in $G$ and so product sets are large.

We now prove the claim by induction. For $n=2$, we can form $W_{2}:=W_{1} *_{2} B_{r_{2}}^{\mathfrak{g}_{2}}(0)$ as $W_{1}=B_{r_{1}}^{\mathfrak{g}_{1}}(0) \subseteq B_{s_{0}}^{\mathfrak{g}_{1}}(0) \subseteq B_{s_{0}}^{\mathfrak{g}_{2}}(0)$ by $(8.4)$, and $B_{r_{2}}^{\mathfrak{g}_{2}}(0) \subseteq B_{s_{0}}^{\mathfrak{g}_{2}}(0)$. Moreover, as $r_{2} \leq s_{0} / 2$, we have $W_{1}+B_{r_{2} / 2}^{\mathfrak{g}_{2}}(0) \subseteq W_{2} \subseteq W_{1}+B_{3 r_{2} / 2}^{\mathfrak{g}_{2}}(0)$ by (8.3). Hence,

$$
B_{r_{1} / 2}^{\mathfrak{g}_{1}}(0)+B_{r_{2} / 2}^{\mathfrak{g}_{2}}(0) \subseteq W_{2} \subseteq B_{3 r_{1} / 2}^{\mathfrak{g}_{1}}(0)+B_{3 r_{2} / 2}^{\mathfrak{g}_{2}}(0)
$$

a fortiori. For the induction step, assume that $n \geq 2$ and that $W_{1}, \ldots, W_{n}$ have already been defined such that (8.5) holds with $k \in\{1, \ldots, n\}$ in place of $n$. In particular, (8.5) holds for $n$ and its right-hand side is a subset of

$$
\sum_{k=1}^{n} B_{s_{0} / 2^{k}}^{\mathfrak{g}_{k}}(0) \subseteq \sum_{k=1}^{n} B_{s_{0} / 2^{k}}^{\mathfrak{g}_{n+1}}(0) \subseteq B_{s_{0}}^{\mathfrak{g}_{n+1}}(0),
$$

using (8.4) for the first inclusion. Thus $W_{n} \subseteq B_{s_{0}}^{\mathfrak{g}_{n+1}}(0)$ and since $r_{n+1} \leq s_{0}$, we deduce that $W_{n+1}:=W_{n} *_{n+1} B_{r_{n+1}}^{\mathfrak{g}_{n+1}}(0)$ can be defined. Moreover,

$$
W_{n}+B_{r_{n+1} / 2}^{\mathfrak{g}_{n+1}}(0) \subseteq W_{n+1} \subseteq W_{n}+B_{3 r_{n+1} / 2}^{\mathfrak{g}_{n+1}}(0),
$$

by (8.3). Using (8.5), we obtain $\sum_{k=1}^{n+1} B_{r_{k} / 2}^{\mathfrak{g}_{k}}(0) \subseteq W_{n+1} \subseteq \sum_{k=1}^{n+1} B_{3 r_{k} / 2}^{\mathfrak{g}_{k}}(0)$, which completes the inductive proof of the claim.

As product sets are large in $G=\bigcup_{n \in \mathbb{N}} G_{n}$ by the preceding, the last and penultimate assertions of the proposition follow from [21, Proposition 11.8] and Theorem 1.1, respectively. 
Acknowledgements I am grateful to my former Ph.D. students Rafael Dahmen and Boris Walter who, in a seminar talk in 2009, sketched a precursor of Lemma 5.1 that feeds into the proof of Theorem 1.2.

\section{References}

[1] S. Albeverio, R. Høegh-Krohn, J. A. Marion, D. H. Testard, and B. S. Torrésani, Noncommutative distributions: unitary representation of gauge groups and algebras. Marcel Dekker, New York, 1993.

[2] A. Arhangel'skii and M. Tkachenko, Topological groups and related structures. World Scientific, Paris, 2008.

[3] T. Banakh, K. Mine, D. Repovš, K. Sakai, and T. Yagasaki, Detecting topological groups which are (locally) homeomorphic to LF-spaces. Topology Appl. 160(2013), 2272-2284. http://dx.doi.org/10.1016/j.topol.2013.07.023

[4] T. Banakh, and D. Repovš, Topological structure of direct limits in the category of uniform spaces. Topology Appl. 157 (2010), 1091-1100. http://dx.doi.org/10.1016/j.topol.2010.01.010

[5] T. Banakh and Y. Yagasaki, Diffeomorphism groups of non-compact manifolds endowed with the Whitney $C^{\infty}$-topology. Topology Appl. 179(2015), 51-61. http://dx.doi.org/10.1016/j.topol.2014.08.015

[6] A. Banyaga, The structure of classical diffeomorphism groups. Kluwer Academic Publishers, Dordrecht, 1997.

[7] A. Bastiani, Applications différentiables et variétés différentiables de dimension infinie. J. Anal. Math. 13(1964), 1-114. http://dx.doi.org/10.1007/BF02786619

[8] W. Bertram, H. Glöckner, and K.-H. Neeb, Differential calculus over general base fields and rings. Expo. Math. 22(2004), 213-282. http://dx.doi.org/10.1016/S0723-0869(04)80006-9

[9] N. Bourbaki, Topological vector spaces. Chapters 1-5, Springer, Berlin, 1987.

[10] _ Lie Groups and Lie algebras. Chapters 1-3, Springer, Berlin, 1989.

[11] P. Cabau and F. Pelletier, Integrability on direct limit Banach manifolds. arxiv:1408.3715v2

[12] R. Dahmen, Analytic mappings between LB-spaces and applications in infinite-dimensional Lie theory. Math. Z. 266(2010), 115-140. http://dx.doi.org/10.1007/s00209-009-0557-0

[13] Regularity in Milnor's sense for ascending unions of Banach-Lie groups. J. Lie Theory 24(2014), 545-560.

[14] S. Dierolf and J. Wengenroth, Inductive limits of topological algebras. In: Linear Topological Spaces and Complex Analysis III. Scientific and Technical Research Council of Turkey (TÜBİTAK), Ankata, 1997, pp. 45-49.

[15] J. Dieudonné, Sur la completion des groupes topologiques. C. R. Acad. Sci. Paris 218(1944), 774-776.

[16] H. Glöckner, Infinite-dimensional Lie groups without completeness restrictions. In: Geometry and analysis on finite- and infinite-dimensional Lie groups. Banach Center Publications 55. Warsaw, 2002, pp. 43-59.

[17] — Lie group structures on quotient groups and universal complexifications for infinite-dimensional Lie groups. J. Funct. Anal. 94(2002), 347-409.

[18] — Lie groups of measurable mappings, Canadian J. Math. 55(2003), 969-999. http://dx.doi.org/10.4153/CJM-2003-039-9

[19] — Fundamentals of direct limit Lie theory. Compositio Math. 141(2005), 1551-1577. http://dx.doi.org/10.1112/S0010437X05001491

[20] Implicit functions from topological vector spaces to Banach spaces. Israel J. Math. 155(2006), 205-252. http://dx.doi.org/10.1007/BF02773955

[21] _ Direct limits of infinite-dimensional Lie groups compared to direct limits in related categories. J. Funct. Anal. 245(2007), 19-61. http://dx.doi.org/10.1016/j.jfa.2006.12.018

[22] — Completeness of locally $k_{\omega}$-groups and related infinite-dimensional Lie groups. Topol. Appl. 228(2017), 277-284. http://dx.doi.org/10.1016/j.topol.2017.05.007

[23] Lie groups over non-discrete topological fields. arxiv:math/0408008

[24] R. S. Hamilton, The inverse function theorem of Nash and Moser. Bull. Amer. Math. Soc. 7(1982), 65-222. http://dx.doi.org/10.1090/S0273-0979-1982-15004-2

[25] E. Hewitt, and K. A. Ross, Abstract harmonic analysis. Vol. 1. Grundlehren der Mathematischen Wissenschaften, 115. Springer-Verlag, Berlin, 1979.

[26] T. Hirai, H. Shimomura, N. Tatsuuma, and E. Hirai, Inductive limits of topologies, their direct products, and problems related to algebraic structures. J. Math. Kyoto Univ. 41(2001), 475-505. http://dx.doi.org/10.1215/kjm/1250517614 
[27] M. W. Hirsch, Differential topology. Springer, New York, 1976.

[28] E. O. Hjelle, and A. Schmeding, Strong topologies for spaces of smooth maps with infinite-dimensional target. Expo. Math. 35(2017), 13-53. http://dx.doi.org/10.1016/j.exmath.2016.07.004

[29] D. C. Hunt and S. A. Morris, Free subgroups of free topological groups. Lect. Notes Math., 372. Springer, Berlin, 1974, pp. 377-387.

[30] S. Illman, The very-strong $C^{\infty}$-topology on $C^{\infty}(M, N)$ and $K$-equivariant maps. Osaka J. Math. 40(2003), 409-428.

[31] J. L. Kelley, General topology. Springer, New York, 1975.

[32] A. Kriegl, and P. W. Michor, The convenient setting of global analysis. Mathematical Surveys and Monographs, 53. American Mathematical Society, Providence, RI, 1997.

[33] P. W. Michor, Manifolds of differentiable mappings. Shiva Publications, Nantwich, 1980.

[34] J. Milnor, Remarks on infinite-dimensional Lie groups. In: Relativity, groups and topology, II. North-Holland, Amsterdam, 1984, pp. 1007-1057.

[35] L. Natarajan, E. Rodríguez-Carrington, and J. A. Wolf, Differentiable structure for direct limit groups. Lett. Math. Phys. 23(1991), 99-109. http://dx.doi.org/10.1007/BF00703721

[36] K.-H. Neeb, Towards a Lie theory of locally convex groups. Jpn. J. Math. 1(2006), 291-468. http://dx.doi.org/10.1007/s11537-006-0606-y

[37] K.-H. Neeb, and F. Wagemann, Lie group structures on groups of smooth and holomorphic maps on non-compact manifolds. Geom. Dedicata 134(2008), 17-60. http://dx.doi.org/10.1007/s10711-008-9244-2

[38] H. Omori, Infinited-dimensional Lie groups. Translations of Mathematical Monographs, 158. American Mathematical Society, Providence, RI, 1997.

[39] A. Pressley and G. Segal, Loop groups. Clarendon Press, Oxford, 1986.

[40] W. Roelcke and S. Dierolf, Uniform structures on topological groups and their quotients. McGraw-Hill, New York, 1981.

[41] J. Schütt, Symmetry groups of principal bundles over non-compact bases. arxiv:1310.8538

[42] N. Tatsuuma, H. Shimomura, and T. Hirai, On group topologies and unitary representations of inductive limits of topological groups and the case of the group of diffeomorphisms. J. Math. Kyoto Univ. 38(1998), 551-578. http://dx.doi.org/10.1215/kjm/1250518067

[43] L. Waelbroeck, Topological vector spaces and algebras. Springer, Berlin, 1971.

[44] J. C. Wells, Invariant manifolds of nonlinear operators. Pacific J. Math. 62(1976), 285-293. http://dx.doi.org/10.2140/pjm.1976.62.285

[45] C. Wockel, Lie group structures on symmetry groups of principal bundles. J. Funct. Anal. 251(2007), 254-288. http://dx.doi.org/10.1016/j.jfa.2007.05.016

[46] A. Yamasaki, Inductive limit of general linear groups. J. Math. Kyoto Univ. 38(1998), 769-779. http://dx.doi.org/10.1215/kjm/1250518008

Institut für Mathematik, Universität Paderborn, Warburger Str. 100, D-33098 Paderborn, Germany e-mail: glockner@math.uni-paderborn.de 